\title{
Low-Complexity Bayesian Estimation of Cluster-Sparse Channels
}

\author{
Tarig Ballal, Tareq Y. Al-Naffouri, Member, IEEE, and Syed Faraz Ahmed
}

\begin{abstract}
This paper addresses the problem of channel impulse response estimation for cluster-sparse channels under the Bayesian estimation framework. We develop a novel low-complexity minimum mean squared error (MMSE) estimator by exploiting the sparsity of the received signal profile and the structure of the measurement matrix. It is shown that due to the banded Toeplitz/circulant structure of the measurement matrix, a channel impulse response, such as underwater acoustic channel impulse responses, can be partitioned into a number of orthogonal or approximately orthogonal clusters. The orthogonal clusters, the sparsity of the channel impulse response and the structure of the measurement matrix, all combined, result in a computationally superior realization of the MMSE channel estimator. The MMSE estimator calculations boil down to simpler in-cluster calculations that can be reused in different clusters. The reduction in computational complexity allows for a more accurate implementation of the MMSE estimator. The proposed approach is tested using synthetic Gaussian channels, as well as simulated underwater acoustic channels. Symbol-error-rate performance and computation time confirm the superiority of the proposed method compared to selected benchmark methods in systems with preamble-based training signals transmitted over clustersparse channels.
\end{abstract}

Index Terms - channel estimation, sparsity, Bayesian, MMSE, underwater acoustics, symbol error rate, Toeplitz/ciculant matrices.

\section{INTRODUCTION}

C HANNEL impulse response estimation is an important wireless communications topic that finds applications in wireless radio communications [1]-[3], underwater acoustic commutations [4]-[6], as well as in SONAR and radar systems $[7,8]$. The literature is rich in methods that can be applied to estimate a channel impulse response. Channel estimation methods can be broadly divided into two groups: data aided (e.g., $[1,9])$ and non data-aided or blind methods (e.g., $[10,11])$. In data aided channel estimation, a (pilot) data sequence that is known to the receiver is transmitted. The receiver leverages this knowledge of the probe signal to estimate the channel from the received

Tarig Ballal is with the Electrical Engineering Department, King Abdullah University of Science and Technology (KAUST), Thuwal 23955, Saudi Arabia. Tareq Y. Al-Naffouri is also with the Electrical Engineering Department, KAUST and the Electrical Engineering Department, King Fahd University of Petroleum and Minerals (KFUPM), Dhahran 3126, Saudi Arabia. Syed Faraz Ahmed is with the Research Institute, KFUPM. e-mail: \{Tarig.Ahmed, Tareq.Alnaffouri\}@kaust.edu.sa \& sfaraz@kfupm.edu.sa. signals. On the other hand, in blind channel estimation methods, the transmitted sequence is unknown to the receiver. In such a case, the receiver needs to rely on more complex signal processing to estimate the channel impulse response from the data. The focus, in this paper, is on the first category-data aided channel estimation.

The transmission of the probe signal through a multipath channel is usually modeled as a linear timeinvariant operation whereby the transmitted sequence is convolved with the channel impulse response (CIR) to produce the received signal [12]. This process is customarily modeled in the discrete-time domain as a matrix-vector multiplication where a so-called measurement matrix, which is derived from the transmitted sequence, multiplies the channel vector to yield the received signal vector. The goal of channel estimation is to recover the CIR from the received data by applying pertinent signal processing operations. A vast number of digital estimation methods are available for CIR estimation. These methods range from simple least squares (LS) based approaches to highly complex Bayesian estimators [12,13].

Bayesian estimation is based on the philosophy that "incorporating the knowledge of the underlying models and prior information into the estimation process results in a class of superior estimators [13].” The knowledge of the statistical distribution of the subject of the estimation process (the CIR in our case) and that of the noise are utilized. Typically, a minimum mean squared error (MMSE) or a maximum a posteriori estimator is produced by the framework. In the recent years, there has been a growing interest in realizing these estimators under signal sparsity conditions [14]-[16]. Adding sparsity to the list is shown to improve performance and also relax the sampling rate requirements if needed. When the signals are sparse it is shown that a model averaging based procedure centred around the signal support can be used to realize the MMSE estimator, and likewise the MAP estimator. However, even with the sparsity assumption, the realization of these estimators is not hassle free. The major issue is the one related to computational complexity that results from the exhaustive nature of these estimators [16]. To reduce the computational complexity involved, additional information needs to be leveraged.

In this paper, we develop a low-complexity Bayesian 
estimator for cluster-sparse channels. Clustered channels are those in which the individual multipath components arrive in groups, thus imposing a clustered pattern into the received signal. Consequently, the received signal vector will be composed of groups of mostly none-zero elements separated by a number of zero elements. Such model arises naturally in some transmission channels such as ultra-wideband [17], OFDM [18] and underwater acoustic channels $[6,19]$, which in addition to the clustering, also exhibit a sparse nature. Herein, we focus on developing a low-complexity MMSE estimator. Following the same route, obtaining the MAP estimator is a straightforward process.

To reduce the computational complexity of the MMSE estimator, we exploit the structure of both the measurement matrix and that of the CIR, i.e., the clustering of the taps of the CIR. The measurement matrix in the channel estimation case has a Toeplitz structure by definition [20]. It will be shown that this form of structure pairs fantastically with the clustered nature of the CIR to allow for reduced-complexity MMSE realization by forming orthogonal clusters of the CIR. Although the MMSE is evaluated in an exhaustive manner (as apposed to greedy approaches that are usually pursued [14]-[16]), the computational complexity is maintained below that of other relevant algorithms. There are three techniques that we use to reduce the computational complexity by leveraging the underlying structures:

1) Orthogonal clustering, which allows us to evaluate the MMSE in a divide-and conquer (and hence parallel) manner (see Eq. (31) further ahead).

2) Recursive calculations of different parameters required for the MMSE evaluation (as in Eq. (34)).

3) Translational invariance, which is a direct result of the Toeplitz structure of the measurement matrix (see Eq. (37)).

\section{A. Organization}

The remainder of this paper is organized as follows. In Section II, the signal model is described. The MMSE channel estimator is discussed in Section III. The proposed low-complexity approach for realizing the MMSE estimator is developed in Section IV and Section V. In Section VI, an algorithm for detecting orthogonally clustered patterns in the received signal is proposed. Simulation results are presented in Section VII, and the conclusions of the paper are given in Section VIII.

\section{B. Nomenclature}

We use lower-case bold-face letters to denote vectors (e.g., $\boldsymbol{y}$ ) and upper-case bold-face letters to denote matrices (e.g., $\boldsymbol{A}$ ). We use upper-case calligraphic font to denote sets (e.g., $\mathcal{S}$ ). A vector symbol with a subscript of a set/index value denotes the corresponding element(s) of the vector (e.g., $\boldsymbol{y}_{i} \equiv \boldsymbol{y}[i]$ and $\boldsymbol{y}_{\mathcal{S}} \equiv$ $\boldsymbol{y}[\mathcal{S}]$ ). Similarly, a matrix symbol with a subscript of a set/index value denotes the matrix/vector comprised of the columns/column specified by the set/index value (e.g., $\boldsymbol{A}_{i} \equiv \boldsymbol{A}[:, i]$ is the $i$ 'th column of $\boldsymbol{A}$, and $\boldsymbol{A}_{\mathcal{S}}$ is the matrix made up of the columns of $\boldsymbol{A}$ whose indices are the elements of the set $\mathcal{S}$ ). Notation such as $\boldsymbol{A}_{i, j}$ denotes the matrix element at the $i$ 'th row and $j$ 'th column of the matrix $\boldsymbol{A}\left(\boldsymbol{A}_{i, j} \equiv \boldsymbol{A}[i, j]\right)^{1}$, while $\mathcal{S}\{i\}$ is the $i$ 'th element of the set. The notations $(.)^{H}$ is used to denote the Hermitian transpose of a vector or matrix. The operation $\mathbb{E}($.$) is used to indicate the statistical$ expectation, while $p($.$) is the probability. Finally, the$ notation $\|.\|_{2}$ denotes the $l^{2}$ (or Euclidean) norm, while |.| is the absolute value of a the real/complex quantities, or the cardinality of a set. The term support (of a vector) is used to refer either to the group of nonzero elements or the corresponding indices of these elements. The term full support refers to the fact that all the elements of the vector or matrix are non-zero.

\section{Signal MODEL}

According to the convolution rule, the received signal can be modeled as a linear combination of scaled and delayed versions of the transmitted signal. In the discrete-time domain, we have the following relationship:

$$
y[n]=\sum_{k=-\infty}^{+\infty} a[n-k] h[k]+v[n],
$$

where $a[n]$ is the $n$ 'th sample of the transmitted signal, $y[n]$ is the corresponding received signal sample, $h[n]$ is the CIR $n$ 'th sample, and $v[n]$ is a sample of noise that is assumed to be additive white Gaussian noise (AWGN) with zero mean and variance $\sigma_{v}^{2}$. Note that the model above ignores frequency dependent effects such as the Doppler phenomenon. The process in (1) can be represented, more conveniently, as

$$
\boldsymbol{y}=\boldsymbol{A} \boldsymbol{h}+\boldsymbol{v},
$$

where $\boldsymbol{y}$ is the received signal (observation) vector, $\boldsymbol{h}$ is the CIR, $\boldsymbol{v}$ is AWGN, and $\boldsymbol{A}$ is the measurement matrix. For the linear system in (2) to have a unique solution without further assumptions, the number of observations (the dimensionality of $\boldsymbol{y}$ ) has to at least be equal to the channel order (the dimensionality of $\boldsymbol{h}$ ). For simplicity, and without loss of generality, we will focus on the equality case, that is $\boldsymbol{y}, \boldsymbol{h}, \boldsymbol{v} \in \mathbb{R}^{N \times 1}$ and $\boldsymbol{A} \in \mathbb{R}^{N \times N}$.

\footnotetext{
${ }^{1}$ These notations for denoting vector and matrix sub-components may not follow the standard notations for vectors and scalars and are used mainly to simplify the presentation of the paper. Descriptions such as $\boldsymbol{A}[:, i]$ are based on Matlab ${ }^{\mathrm{TM}}$ programming language.
} 


\section{A. Measurement Matrix Structure}

The structure of the measurement matrix, based on (1) and (2), takes the form

$$
\boldsymbol{A}=\left[\begin{array}{ccccc}
a_{0} & a_{-1} & a_{-2} & \cdots & a_{-N+1} \\
a_{1} & a_{0} & a_{-1} & \cdots & a_{-N+2} \\
a_{2} & a_{1} & a_{0} & \cdots & a_{-N+3} \\
\vdots & \vdots & \vdots & \ddots & \vdots \\
a_{N-1} & a_{N-2} & a_{N-3} & \cdots & a_{0}
\end{array}\right],
$$

where $a_{n}$ is used to represent $a[n]$ in (1). Note that the elements of $\boldsymbol{A}$ satisfy the property $\boldsymbol{A}_{i, j}=$ $\boldsymbol{A}_{i+1, j+1}, \forall i, j \in\{0, \cdots, N-1\}$, which makes $\boldsymbol{A}$ a Toeplitz matrix. Normally, the transmission time is much shorter than the observation time (to allow the receiver to collect various multipath components). Therefore, $\boldsymbol{a}_{n}$ is zero except for its first few elements. Let the support of $\boldsymbol{a}_{n}$ be confined to the indices $n \in\{0, \cdots, L-1\}$. This leads to a banded matrix structure of $\boldsymbol{A}$ with bandwidth equal to $L$, as shown in Fig. 1.

To simplify the computations and exposition, it is desirable that the norm of each column of $\boldsymbol{A}$ has the same value. For a Toeplitz structure, this feature cannot be guaranteed in many cases including the square matrix case. Namely, the last few columns may have a smaller number of active elements, and hence a smaller norm, than the rest of the columns. These columns will then have to be treated separately. However, to simplify the presentation, we artificially impose equal norm on all the matrix columns. This is achieved by modifying $\boldsymbol{A}$ into a circulant structure by making $\boldsymbol{A}$ satisfy the same condition of the Toeplitz matrix $\left(\boldsymbol{A}_{i, j}=\boldsymbol{A}_{i+1, j+1}, \forall i, j \in\{0, \cdots, N-1\}\right)$, but with $i+1$ and $j+1$ taken modulo $N$. This modification of $\boldsymbol{A}$ requires judicious addition of a few non-zero values to specific top-right elements of $\boldsymbol{A}$ such that each column contains the same non-zero values taken at different shifts. Fig 1 depicts an illustration of the measurement matrix; the top-right triangle marks the elements that were altered in order to induce the circulant-matrix structure (without these elements, $\boldsymbol{A}$ is just a banded Toeplitz matrix). This modification of the matrix is justified as follows:

- This circulant structure arises naturally in the case where the transmission is repeated in a periodic manner, or in systems using techniques such as OFDM [1].

- In the case of non-periodic transmission, the modification happens only in the last columns. If the observation time is sufficiently large (large $N)$ that multipath is reasonably attenuated, or completely dies out, for channel taps $\{N-L-$ $1, \cdots, N-1\}$, the matrix modification will not have any effect on the validity of (2). These introduced values will cancel out when multiplied by

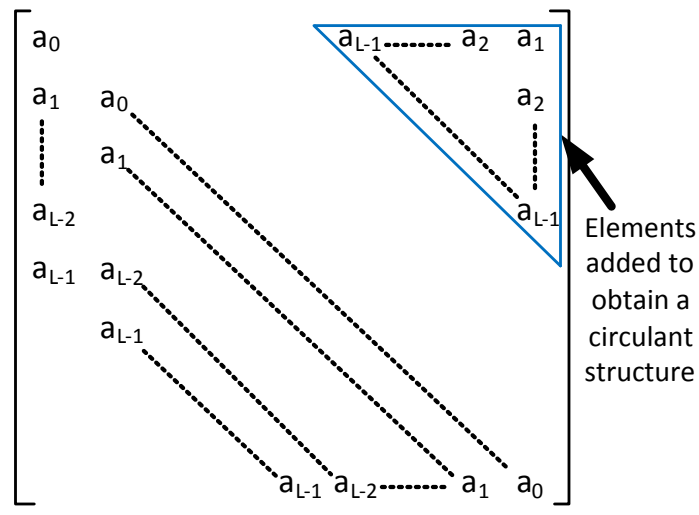

Figure 1: Illustration of the structure of the measurement matrix $\boldsymbol{A}$ showing only the non-zero elements of the matrix. The horizontal and vertical doted lines indicates increment or decrement of order, while inclined lines indicate the repetition of the same value.

the corresponding zero, or approximately zero, elements of $\boldsymbol{h}$ at the indices $\{N-L-1, \cdots, N-1\}$. For example, by partitioning the matrix/vector, we obtain the following result

$$
\begin{aligned}
\boldsymbol{A} \boldsymbol{h} & =\left[\begin{array}{ll}
\boldsymbol{A}_{0} & \mathbf{0} \\
\boldsymbol{A}_{1} & \boldsymbol{A}_{2}
\end{array}\right]\left[\begin{array}{c}
\boldsymbol{h}_{0} \\
\mathbf{0}
\end{array}\right]=\left[\begin{array}{ll}
\boldsymbol{A}_{0} & \boldsymbol{A}_{c} \\
\boldsymbol{A}_{1} & \boldsymbol{A}_{2}
\end{array}\right]\left[\begin{array}{c}
\boldsymbol{h}_{0} \\
\mathbf{0}
\end{array}\right] \\
& =\left[\begin{array}{l}
\boldsymbol{A}_{0} \boldsymbol{h}_{0} \\
\boldsymbol{A}_{1} \boldsymbol{h}_{0}
\end{array}\right]
\end{aligned}
$$

where $\boldsymbol{A}_{c}$ contains non-zero elements that make $\boldsymbol{A}$ a circulant matrix. Throughout this paper, it will be assumed that (2) is both valid and accurate when $\boldsymbol{A}$ is altered to have a circulant structure.

\section{B. CIR Structure}

In this work, the CIR is assumed to consist of clusters separated by zero taps. The clusters are dominantly non-zero valued but may have some zero taps in between. This can be modeled as

$$
\boldsymbol{h}=\boldsymbol{g} \odot \boldsymbol{b},
$$

where $\boldsymbol{g}$ is a vector with full support (all non-zero elements), $\boldsymbol{b}$ is a binary vector that represents the support of $\boldsymbol{h}$, and $\odot$ denotes element-by-element multiplication.

\section{BAYESIAN CHANNEL ESTIMATION}

The MMSE CIR estimator, based on the model in (2), can be formed as $[14,16]$

$$
\hat{\boldsymbol{h}}_{\mathrm{MMSE}}=\sum_{\mathcal{S}=\boldsymbol{\Omega}_{0}}^{\boldsymbol{\Omega}_{M-1}} p(\mathcal{S} \mid \boldsymbol{y}) \mathbb{E}(\boldsymbol{h} \mid \boldsymbol{y}, \mathcal{S}),
$$

where the sum is performed over all possible support sets $\mathcal{S} \in\left\{\boldsymbol{\Omega}_{0}, \cdots, \boldsymbol{\Omega}_{M-1}\right\}$, where $M=\sum_{k=0}^{N}\left(\begin{array}{c}N \\ k\end{array}\right)=$ 
$2^{N}$ (i.e., all subsets of the set $\{1, \cdots, N\}$ ). To evaluate (5), we need to find the a posteriori expected value of the amplitudes conditioned on the support $(\mathbb{E}(\boldsymbol{h} \mid \boldsymbol{y}, \mathcal{S}))$, weighted by the a posteriori probability of the support $(p(\mathcal{S} \mid \boldsymbol{y}))$ for each individual support, and average the results for all the $M$ support cases. By using Bayes' rule the a posteriori probability can be expressed as

$$
p(\mathcal{S} \mid \boldsymbol{y})=\frac{p(\boldsymbol{y}, \mathcal{S})}{p(\boldsymbol{y})}=\frac{p(\boldsymbol{y} \mid \mathcal{S}) p(\mathcal{S})}{p(\boldsymbol{y})}
$$

Substituting (6) into (5) results in the MMSE estimator

$$
\hat{\boldsymbol{h}}_{\mathrm{MMSE}}=\frac{1}{p(\boldsymbol{y})} \sum_{\mathcal{S}=\boldsymbol{\Omega}_{0}}^{\boldsymbol{\Omega}_{M-1}} p(\boldsymbol{y} \mid \mathcal{S}) p(\mathcal{S}) \mathbb{E}(\boldsymbol{h} \mid \boldsymbol{y}, \mathcal{S}),
$$

where $p(\boldsymbol{y})$ is calculated as

$$
p(\boldsymbol{y})=\sum_{\mathcal{S}=\boldsymbol{\Omega}_{0}}^{\boldsymbol{\Omega}_{M-1}} p(\boldsymbol{y} \mid \mathcal{S}) p(\mathcal{S}) .
$$

Note that the MMSE estimator in (5) attempts to estimate all zero and non-zero elements of $\boldsymbol{h}$. However, it is sufficient to estimate the elements that coincide with the support of $\boldsymbol{h}$. This is the approach that will be pursued in the following sections.

From Eq. (7), it appears that to evaluate the MMSE CIR estimator, we need to compute the following three terms:

1) the a priori probability support $p(\mathcal{S})$;

2) the likelihood $p(\boldsymbol{y} \mid \mathcal{S})$; and

3) the a posteriori expectation $\mathbb{E}(\boldsymbol{h} \mid \boldsymbol{y}, \mathcal{S})$.

In the following subsections, we show how to compute each element of this triad.

\section{A. Computing The a Priori Probability $p(\mathcal{S})$}

The a priori probability of a certain support $p(\mathcal{S})$ cannot be known precisely. Thus, we resort to approximating this probability as follows. First, we model the arrival of a multipath component during a sample duration as a Bernoulli trial with probability of success $p_{b}$. This means that the probability of a certain CIR tap to be non-zero is equal to $p_{b}$. Consequently, the probability that the taps of the indices given by $\mathcal{S}$ are active (or non-zero) can be written as

$$
p(\mathcal{S})=p_{b}^{|\mathcal{S}|}\left(1-p_{b}\right)^{N-|\mathcal{S}|},
$$

Note that the Bernoulli probability $p_{b}$ can be related to the sparsity rate of the CIR. In fact, the two quantities are equivalent.

\section{B. Computing The Likelihood $p(\boldsymbol{y} \mid \mathcal{S})$}

The hurdle on the way of finding the likelihood associated with a certain support is that the probability distribution of the amplitudes of $\boldsymbol{y}$ is either unknown or non-Gaussian. Both situations make it difficult to estimate $p(\boldsymbol{y} \mid \mathcal{S})$. However, given the support $\mathcal{S}$, we can say that $\boldsymbol{y}$ is made up of a vector that lies in the subspace spanned by the columns of a matrix $\boldsymbol{A}_{\mathcal{S}}$ plus an AWGN vector $\boldsymbol{v}$, where $\boldsymbol{A}_{\mathcal{S}}$ is the matrix that consists only of the columns of $\boldsymbol{A}$ whose indices are given by $\mathcal{S}$. Mathematically speaking,

$$
\boldsymbol{y}=\boldsymbol{A}_{\mathcal{S}} \boldsymbol{h}_{\mathcal{S}}+\boldsymbol{v} .
$$

Now, consider the projection matrix, $\boldsymbol{P}_{\mathcal{S}}^{\perp}$, that projects onto the orthogonal complement subspace of $\boldsymbol{A}_{\mathcal{S}}$ and that is given by

$$
\boldsymbol{P}_{\mathcal{S}}^{\perp}=\boldsymbol{I}-\boldsymbol{A}_{\mathcal{S}} \boldsymbol{B}_{\mathcal{S}}^{-1} \boldsymbol{A}_{\mathcal{S}}^{H},
$$

where $\boldsymbol{B}_{\mathcal{S}} \triangleq \boldsymbol{A}_{\mathcal{S}}^{H} \boldsymbol{A}_{\mathcal{S}}$. The projection $\boldsymbol{P}_{\mathcal{S}}^{\perp} \boldsymbol{y}$ will be free of any contribution of $\boldsymbol{h}$ and hence it, approximately, has a Gaussian distribution (due to the AWGN). In other words, we have $\boldsymbol{P}_{\mathcal{S}}^{\perp} \boldsymbol{y} \approx \boldsymbol{P}_{\mathcal{S}}^{\perp} \boldsymbol{v}$ (see the discussion in [16]). Consequently the likelihood $p(\boldsymbol{y} \mid \mathcal{S})$ can be approximated up to an irrelevant proportionality constant as

$$
p(\boldsymbol{y} \mid \mathcal{S}) \propto \exp \left(-\frac{1}{2 \sigma_{v}^{2}}\left\|\boldsymbol{P}_{\mathcal{S}}^{\perp} \boldsymbol{y}\right\|_{2}^{2}\right) .
$$

For a detailed derivation of (12), the reader is referred to [16]. Since the proportionality constant will appear in both $p(\boldsymbol{y} \mid \mathcal{S})$ and $p(\boldsymbol{y})$ of (7), we can disregard this proportionality constant and write

$$
p(\boldsymbol{y} \mid \mathcal{S}) \approx \exp \left(-\frac{1}{2 \sigma_{v}^{2}}\left\|\boldsymbol{P}_{\mathcal{S}}^{\perp} \boldsymbol{y}\right\|_{2}^{2}\right) .
$$

\section{Computing The a Posteriori Expectation $\mathbb{E}(\boldsymbol{h} \mid \boldsymbol{y}, \mathcal{S})$}

Again, due to the non-Gaussian statistics of $\boldsymbol{h}$, the expectation $\mathbb{E}(\boldsymbol{h} \mid \boldsymbol{y}, \mathcal{S})$ cannot be evaluated in a straightforward manner. Therefore, we resort to approximating it by the projection

$$
\begin{aligned}
\mathbb{E}\left(\boldsymbol{h}_{\mathcal{S}} \mid \boldsymbol{y}, \mathcal{S}\right) & \approx\left(\boldsymbol{A}_{\mathcal{S}}^{H} \boldsymbol{A}_{\mathcal{S}}\right)^{-1} \boldsymbol{A}_{\mathcal{S}}^{H} \boldsymbol{y} \\
& =\boldsymbol{B}_{\mathcal{S}}^{-1} \boldsymbol{q}_{\mathcal{S}},
\end{aligned}
$$

where $\boldsymbol{q} \triangleq \boldsymbol{A}^{H} \boldsymbol{y}$ and the subscript $\mathcal{S}$ indicates which entries of $\boldsymbol{q}$ are kept. The approximation in (14) coincides with the least squares (LS) estimator of $\boldsymbol{h}_{\mathcal{S}}$. For the AWGN case, this is also the best linear unbiased estimator (BLUE) [13]. Note that in (14) we focus only on the non-zero part of the CIR, and therefore, $h_{\mathcal{S}}$ has replaced $\boldsymbol{h}$ in the expectation expression.

So far, we have explained ways for computing or approximating the three terms required for evaluating the contributions of each support to the CIR MMSE estimator. The major problem is, however, the tremendous computational complexity involved in evaluating these terms for all the $M=2^{N}$ support sets, which makes the MMSE estimator in (7) impractical. In the subsequent sections, we show how the structure of the measurement matrix together with the clustersparse nature of the CIR can be used to reduce the 
computational complexity to practical levels.

\section{Reducing The Complexity of The MMSE Estimator USING ORTHOgONAL CLUSTERING}

To reduce the complexity associated with the evaluation of the MMSE estimator in (7), we exploit the following two structures:

- The structure of the CIR, i.e., the fact that the CIR is cluster-sparse; and

- The Toeplitz/circulant structure of the measurement matrix.

Knowledge of these two structures is leveraged to evaluate (7) in a "divide and conquer" manner. Namely, we divide $\boldsymbol{h}$ into orthogonal clusters and break down the MMSE estimator in (7) into a number of significantly "smaller" MMSE estimators. The measurement matrix structure is further utilized to reduce the computational complexity even more by reusing the computations of one cluster in the other clusters.

\section{A. Orthogonal Clustering (OC)}

Let us, again, consider the signal model in (2) and the measurement matrix depicted in Fig. 1. The matrix $\boldsymbol{A}$ has a banded circulant structure with a bandwidth equal to $L$. In practice, the duty cycle of the probe signal transmission period is very small. In other words, the actual transmission of the probe signal takes place over a short period of time compared to the silence period that follows the probe signal and that is required for collecting the multipath arrivals, without interfering with the data transmitted in the next time slot. This results in the dimension of the matrix $\boldsymbol{A}$ satisfying

$$
N \gg l \text {. }
$$

For any banded Toeplitz or circulant matrix, it can easily be shown that any pair of columns, $\boldsymbol{A}_{i}$ and $\boldsymbol{A}_{j}$, which are separated by a column separation of $\beta_{i j} \geq L$, are perfectly orthogonal, i.e.,

$$
\boldsymbol{A}_{i}^{H} \boldsymbol{A}_{j}=0 ; \forall i, j \in\{0, \cdots, N-1\} \quad \text { if } \quad \beta_{i j} \geq L .
$$

Note that for a Toeplitz matrix, $\beta_{i j}$ is simply given by $\beta_{i j}=|i-j|$. For a circulant matrix, the circulant structure (i.e., the continuity between the first and last column) should be taken into account. In this case, $\beta_{i j}$ is given by

$$
\beta_{i j}=\min (|i-j|, N-|i-j|) .
$$

The property in (16) can easily be extended to sub-matrices. Consider a pair of sub-matrices of $\boldsymbol{A}$ denoted by $\boldsymbol{A}_{\mathcal{O}_{i}}$ and $\boldsymbol{A}_{\mathcal{O}_{j}}$, where the support sets $\mathcal{O}_{i}$ and $\mathcal{O}_{j}$ are non-overlapping and non-interleaving, i.e., $\max \left(\mathcal{O}_{i}\right)<\min \left(\mathcal{O}_{j}\right)$. In this case, we have the following more general version of orthogonality:

$$
\boldsymbol{A}_{\mathcal{O}_{i}}^{H} \boldsymbol{A}_{\mathcal{O}_{j}}=\mathbf{0} \quad \text { if } \quad \beta_{i j} \geq L
$$

where $\beta_{i j}$ is defined here as

$$
\beta_{i j}=\min \left(\mathcal{O}_{j}\right)-\max \left(\mathcal{O}_{i}\right) .
$$

Now, let us look at the CIR which consists of sparse clusters. A pair of these clusters can be orthogonal, depending on whether the sub-matrices of $\boldsymbol{A}$, which interact with these clusters, are orthogonal or not (see Eq. (18)). Fig. 2 shows an example with a $16 \times 16$ measurement matrix and a CIR with two orthogonal clusters. The probe signal has $L=5$ samples, which makes $\mathcal{O}_{0}$ and $\mathcal{O}_{1}$ perfectly orthogonal since $\beta_{01}=$ $10-4=6>L$.

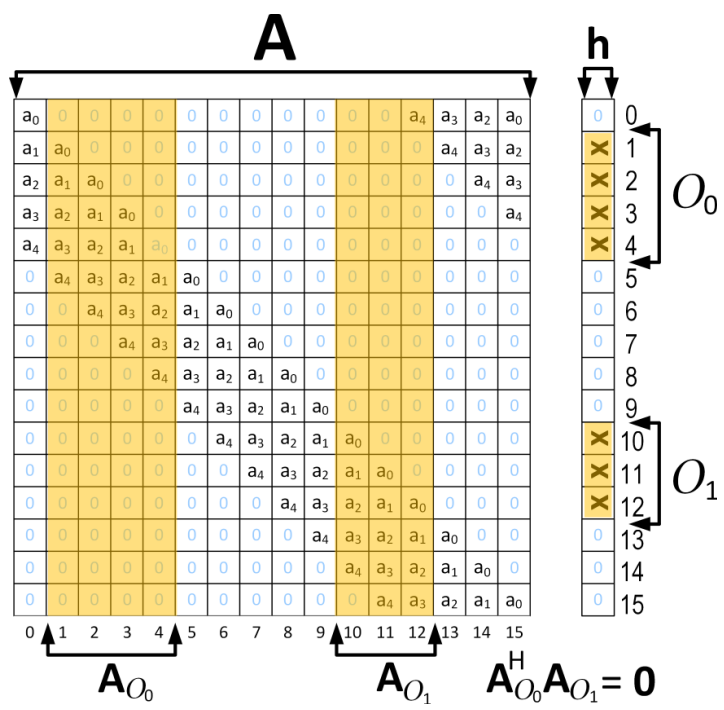

Figure 2: An exemplar layout of the structures of a CIR, $h$, with two orthogonal clusters; and a $16 \times 16$ measurement matrix, $\boldsymbol{A}$, with $L=5$ and $\beta_{01}=6$.

Let us now assume that $\boldsymbol{h}$ can be divided into $C$ pairwise orthogonal clusters as in (18). We will denote the support set corresponding to each cluster by $\mathcal{O}_{c}, \forall c=0, \cdots, C-1$. The total support is thus given by

$$
\mathcal{O}=\bigcup_{c=0}^{C-1} \mathcal{O}_{c}
$$

\section{B. Computation Reduction by $O C$}

In this subsection, we show how orthogonal clustering can help reduce the computational complexity of the MMSE estimator in (7). To do so, in performing the sum (7), we consider only the supports $\mathcal{S}$ that are confined to the orthogonal clusters. Consequently, the MMSE estimation in (7) is reduced to estimating the non-zero elements of $\boldsymbol{h}$,

$$
\hat{\boldsymbol{h}}_{\mathcal{O}}=\frac{\sum_{\mathcal{S} \subseteq \mathcal{O}} p(\boldsymbol{y} \mid \mathcal{S}) p(\mathcal{S}) \mathbb{E}(\boldsymbol{h} \mid \boldsymbol{y}, \mathcal{S})}{\sum_{\mathcal{S} \subseteq \mathcal{O}} p(\boldsymbol{y} \mid \mathcal{S}) p(\mathcal{S})},
$$

where the sum is performed over all the $M$ possible support sets $\mathcal{S} \subseteq \mathcal{O}$ with $M$ redefined as $M=$ 
$\sum_{k=1}^{|\mathcal{O}|}\left(\begin{array}{c}|\mathcal{O}| \\ k\end{array}\right)$

In this case, each support set can be represented as the union of $C$ subsets, each subset coming from a different cluster, i.e.,

$$
\mathcal{S}=\bigcup_{c=0}^{C-1} \mathcal{S}_{c}, \mathcal{S}_{c} \subseteq \mathcal{O}_{c}
$$

where $\mathcal{S}_{c}$ is allowed to be empty, i.e., $\mathcal{S}_{c}=\{\}$. Now, consider the matrices $\boldsymbol{A}_{\mathcal{S}_{c}}, c=0, \cdots, C-1$, which are pairwise orthogonal in the sense reflected by Eq. (18). The support matrix, $\boldsymbol{A}_{\mathcal{S}}$, can be written in the form

$$
\boldsymbol{A}_{\mathcal{S}}=\left[\boldsymbol{A}_{\mathcal{S}_{0}}, \ldots, \boldsymbol{A}_{\mathcal{S}_{C-1}}\right] \text {. }
$$

Subsequently, it will be shown how this structure of $\boldsymbol{A}_{\mathcal{S}}$ can be used to make our computation of (21) more efficient.

1) A posteriori expectation: Let us start with the a posteriori expectation in Eq. (14). The pairwise orthogonality of $\boldsymbol{A}_{\mathcal{S}_{c}}$ and Eq. (23) allows us to write the inverse term $\boldsymbol{B}_{\mathcal{S}}^{-1}=\left(\boldsymbol{A}_{\mathcal{S}}^{H} \boldsymbol{A}_{\mathcal{S}}\right)^{-1}$ in a block diagonal matrix form

$$
\boldsymbol{B}_{\mathcal{S}}^{-1}=\left[\begin{array}{cccc}
\boldsymbol{B}_{\mathcal{S}_{0}}^{-1} & 0 & \cdots & 0 \\
0 & \boldsymbol{B}_{\mathcal{S}_{1}}^{-1} & \cdots & 0 \\
\vdots & \vdots & \ddots & \vdots \\
0 & 0 & 0 & \boldsymbol{B}_{\mathcal{S}_{C-1}}^{-1}
\end{array}\right]
$$

where $\boldsymbol{B}_{\mathcal{S}_{c}} \triangleq\left(\boldsymbol{A}_{\mathcal{S}_{c}}^{H} \boldsymbol{A}_{\mathcal{S}_{c}}\right), \forall c=0, \cdots, C-1$ is an $\left|S_{c}\right| \times\left|S_{c}\right|$ matrix.

Now, let us consider the vector $\boldsymbol{q}_{\mathcal{S}} \triangleq \boldsymbol{A}_{\mathcal{S}}^{H} \boldsymbol{y}$. Using (23), we can write $\boldsymbol{q}_{\mathcal{S}}$ in the form

$$
\boldsymbol{q}_{\mathcal{S}}=\left[\begin{array}{c}
\boldsymbol{A}_{\mathcal{S}_{0}}^{H} \boldsymbol{y} \\
\boldsymbol{A}_{\mathcal{S}_{1}}^{H} \boldsymbol{y} \\
\vdots \\
\boldsymbol{A}_{\mathcal{S}_{C-1}^{H}}^{H} \boldsymbol{y}
\end{array}\right]=\left[\begin{array}{c}
\boldsymbol{q}_{\mathcal{S}_{0}} \\
\boldsymbol{q}_{\mathcal{S}_{1}} \\
\vdots \\
\boldsymbol{q}_{\mathcal{S}_{C-1}}
\end{array}\right]
$$

By substituting (24) and (25) into (14), we obtain

$$
\begin{aligned}
& \mathbb{E}\left(\boldsymbol{h}_{\mathcal{S}} \mid \boldsymbol{y}, \mathcal{S}\right) \approx \\
& {\left[\begin{array}{c}
\boldsymbol{B}_{\mathcal{S}_{0}}^{-1} \boldsymbol{q}_{\mathcal{S}_{0}} \\
\boldsymbol{B}_{\mathcal{S}_{1}}^{-1} \boldsymbol{q}_{\mathcal{S}_{1}} \\
\vdots \\
\boldsymbol{B}_{\mathcal{S}_{C-1}^{-1}} \boldsymbol{q}_{\mathcal{S}_{C-1}}
\end{array}\right]=\left[\begin{array}{c}
\mathbb{E}\left(\boldsymbol{h}_{\mathcal{S}_{0}} \mid \boldsymbol{y}, \mathcal{S}_{0}\right) \\
\mathbb{E}\left(\boldsymbol{h}_{\mathcal{S}_{1}} \mid \boldsymbol{y}, \mathcal{S}_{1}\right) \\
\vdots \\
\mathbb{E}\left(\boldsymbol{h}_{\mathcal{S}_{C-1}} \mid \boldsymbol{y}, \mathcal{S}_{C-1}\right)
\end{array}\right] .}
\end{aligned}
$$

In (26), the computation of the expectation $\mathbb{E}\left(\boldsymbol{h}_{\mathcal{S}} \mid \boldsymbol{y}, \mathcal{S}\right)$ is divided into the computation of $C$ smaller expectations, $\mathbb{E}\left(\boldsymbol{h}_{\mathcal{S}} \mid \boldsymbol{y}, \mathcal{S}_{c}\right) \in \mathbb{R}^{\left|\mathcal{S}_{c}\right| \times 1}$. Each expectation computation in (26) requires the inversion of a considerably smaller matrix $\boldsymbol{B}_{\mathcal{S}_{c}}$ compared to the full support matrix $\boldsymbol{B}_{\mathcal{S}}$ in (14), hence reducing the computational complexity involved.

2) Likelihood: Now let us turn our attention to the likelihood probability $p(\boldsymbol{y} \mid \mathcal{S})$ given by (13). To evaluate this probability, we firstly need to compute $\boldsymbol{P}_{\mathcal{S}}^{\perp}$ as in (11). Using (23) and (24), (11) can be broken down in a similar manner to (26). This yields

$$
\begin{aligned}
\boldsymbol{P}_{\mathcal{S}}^{\perp} & =\boldsymbol{I}-\sum_{c=1}^{C-1} \boldsymbol{A}_{\mathcal{S}_{c}} \boldsymbol{B}_{\mathcal{S}_{c}}^{-1} \boldsymbol{A}_{\mathcal{S}_{c}}^{H} \\
& =-(C-1) \boldsymbol{I}+\sum_{c=1}^{C-1} \boldsymbol{P}_{\mathcal{S}_{c}}^{\perp}
\end{aligned}
$$

where $\boldsymbol{P}_{\mathcal{S}_{c}}^{\perp} \triangleq \boldsymbol{A}_{\mathcal{S}_{c}} \boldsymbol{B}_{\mathcal{S}_{c}}^{-1} \boldsymbol{A}_{\mathcal{S}_{c}}^{H} \in \mathbb{R}^{N \times N}$. It is easy to see that, due to the pairwise orthogonality of the submatrices $\left\{\boldsymbol{A}_{\mathcal{S}_{c}}\right\},\left\{\boldsymbol{P}_{\mathcal{S}_{c}}^{\perp}\right\}$ are also pairwise orthogonal. This, together with the idempotent property of the projection matrix, allows us to write

$$
\left\|\boldsymbol{P}_{\mathcal{S}}^{\perp} \boldsymbol{y}\right\|_{2}^{2}=-(C-1)\|\boldsymbol{y}\|_{2}^{2}+\sum_{c=1}^{C-1}\left\|\boldsymbol{P}_{\mathcal{S}_{c}}^{\perp} \boldsymbol{y}\right\|_{2}^{2} .
$$

By plugging (28) into (13) and then (21), we realize the following. The first term in (28) is irrelevant since it contributes equally to both the numerator and denominator of (21). Hence, this term cancels out when $p(\boldsymbol{y} \mid \mathcal{S})$ is substituted in (21). By ignoring this term and substituting (28) in (13), it is easy to show that

$$
p(\boldsymbol{y} \mid \mathcal{S}) \approx \prod_{c=1}^{C-1} p\left(\boldsymbol{y} \mid \mathcal{S}_{c}\right)
$$

where $p\left(\boldsymbol{y} \mid \mathcal{S}_{c}\right)$ is obtained from (13) by replacing $\mathcal{S}$ with $\mathcal{S}_{c}$.

3) A Priori Probability: This probability (see Eq. (9)) can be expressed simply as

$$
\begin{aligned}
p(\mathcal{S}) & =p_{b}^{\left(\sum_{c=1}^{C-1}\left|\mathcal{S}_{c}\right|\right)}\left(1-p_{b}\right)^{\left(N-\sum_{c=1}^{C-1}\left|\mathcal{S}_{c}\right|\right)} \\
& =\prod_{c=1}^{C-1} p\left(\mathcal{S}_{c}\right)
\end{aligned}
$$

4) Final MMSE Estimator: Substituting (26), (29) and (30) into (21), we obtain

$$
\begin{aligned}
& \hat{\boldsymbol{h}}_{\mathcal{O}}= \\
& {\left[\begin{array}{c}
\frac{\sum_{\mathcal{S} \subseteq \mathcal{O}_{0},} p(\boldsymbol{y} \mid \mathcal{S}) p(\mathcal{S}) \mathbb{E}\left(\boldsymbol{h}_{\mathcal{S}} \mid \boldsymbol{y}, \mathcal{S}\right)}{p_{0}} \\
\frac{\sum_{\mathcal{S} \subseteq \mathcal{O}_{1}} p(\boldsymbol{y} \mid \mathcal{S}) p(\mathcal{S}) \mathbb{E}\left(\boldsymbol{h}_{\mathcal{S}} \mid \boldsymbol{y}, \mathcal{S}\right)}{p_{1}} \\
\vdots \\
\frac{\sum_{\mathcal{S} \subseteq \mathcal{O}_{C-1}} p(\boldsymbol{y} \mid \mathcal{S}) p(\mathcal{S}) \mathbb{E}\left(\boldsymbol{h}_{\mathcal{S}} \mid \boldsymbol{y}, \mathcal{S}\right)}{p_{C-1}}
\end{array}\right],}
\end{aligned}
$$

where $p_{c} \triangleq \sum_{\mathcal{S}_{c} \subset \mathcal{O}_{c}} p\left(\boldsymbol{y} \mid \mathcal{S}_{c}\right) p\left(\mathcal{S}_{c}\right)$ (see Appendix A for a proof). In (31), each summation that pertains to a cluster, say cluster $c$, is performed over all the $M_{c}$ possible support sets for that cluster, where $M_{c}=\sum_{k=0}^{\left|\mathcal{O}_{c}\right|}\left(\begin{array}{c}\left|\mathcal{O}_{c}\right| \\ k\end{array}\right)=2^{\left|\mathcal{O}_{c}\right|}$ is the total number of support sets for cluster $c$. Note that (31) gives a channel gain estimate at the support of the CIR. The actual CIR 
estimate ( $\left.\hat{\boldsymbol{h}}_{\mathrm{MMSE}}\right)$ can easily be obtained from $\hat{\boldsymbol{h}}_{\mathcal{O}}$ by positioning the clusters at their corresponding delay locations.

\section{Further Computational Complexity REDUCTION}

Having reduced the MMSE estimate to the clusterwise computations in Eq. (31), in this section, we propose ways of reducing the computational complexity of (31) even further. The MMSE form in (31) has been reached through the incorporation of the orthogonal clustering facility of the CIR when projected on the circulant matrix structure. The computational reduction proposed in this section is mainly due to the measurement matrix structure.

\section{A. In-Cluster Recursive Computations}

Let us investigate how the two data-dependent quantities involved (the likelihood and the expectation) can be computed in an order recursive manner. This is a result of the fact that in evaluating any of the summations in (31), we have sets of $\mathcal{S}$ 's that are increments of each other.

The core in the computation of the likelihood, according to (13), is the quantity $\left\|\boldsymbol{P}_{\mathcal{S}}^{\perp} \boldsymbol{y}\right\|_{2}^{2}$, which can be related to the expectation as follows:

$$
\begin{aligned}
\left\|\boldsymbol{P}_{\mathcal{S}}^{\perp} \boldsymbol{y}\right\|_{2}^{2} & =\|\boldsymbol{y}\|_{2}^{2}-\boldsymbol{y}^{H} \boldsymbol{A}_{\mathcal{S}} \boldsymbol{B}_{\mathcal{S}}^{-1} \boldsymbol{A}_{\mathcal{S}}^{H} \boldsymbol{y} \\
& =\|\boldsymbol{y}\|_{2}^{2}-\boldsymbol{y}^{H} \boldsymbol{A}_{\mathcal{S}} \mathbb{E}\left(\boldsymbol{h}_{\mathcal{S}} \mid \boldsymbol{y}, \mathcal{S}\right) \\
& =\|\boldsymbol{y}\|_{2}^{2}-\boldsymbol{q}_{\mathcal{S}}^{H} \mathbb{E}\left(\boldsymbol{h}_{\mathcal{S}} \mid \boldsymbol{y}, \mathcal{S}\right)
\end{aligned}
$$

where the last line is obtained by substituting $\boldsymbol{q}_{\mathcal{S}}=$ $\boldsymbol{A}_{\mathcal{S}}^{H} \boldsymbol{y}$. The quantity $\left\|\boldsymbol{P}_{\mathcal{S}}^{\perp} \boldsymbol{y}\right\|_{2}^{2}$ represents a scaled version of the log-likelihood. As such, the recursive implementation of the MMSE depends heavily on the recursive implementation of the expectation, $\mathbb{E}\left(\boldsymbol{h}_{\mathcal{S}} \mid \boldsymbol{y}, \mathcal{S}\right)$.

Now, consider a support set $\mathcal{S}$. Let us write this support set as a concatenation of a subset $\underline{\mathcal{S}}$ and an incremental index $i$, i.e., $\mathcal{S}=\underline{\mathcal{S}} \cup\{i\}$. Based on this way of expressing the support $\mathcal{S}$, the corresponding expectation can be evaluated as

$$
\begin{aligned}
\mathbb{E}\left(\boldsymbol{h}_{\mathcal{S}} \mid \boldsymbol{y}, \mathcal{S}\right) & =\left(\boldsymbol{A}_{\mathcal{S}}^{H} \boldsymbol{A}_{\mathcal{S}}\right)^{-1} \boldsymbol{A}_{\mathcal{S}}^{H} \boldsymbol{y} \\
& =\left(\left[\begin{array}{l}
\boldsymbol{A}_{\underline{\mathcal{S}}}^{H} \\
\boldsymbol{A}_{i}^{H}
\end{array}\right]\left[\begin{array}{ll}
\boldsymbol{A}_{\underline{\mathcal{S}}} & \boldsymbol{A}_{i}
\end{array}\right]\right)^{-1}\left[\begin{array}{c}
\boldsymbol{A}_{\underline{\mathcal{S}}}^{H} \boldsymbol{y} \\
\boldsymbol{A}_{i}^{H} \boldsymbol{y}
\end{array}\right] \\
& =\left[\begin{array}{ll}
\boldsymbol{A}_{\underline{\mathcal{S}}}^{H} \boldsymbol{A}_{\underline{\mathcal{S}}} & \boldsymbol{A}_{\underline{\mathcal{S}}}^{H} \boldsymbol{A}_{i} \\
\boldsymbol{A}_{i}^{H} \boldsymbol{A}_{\underline{\mathcal{S}}} & \boldsymbol{A}_{i}^{H} \boldsymbol{A}_{i}
\end{array}\right]^{-1}\left[\begin{array}{c}
\boldsymbol{A}_{\underline{\mathcal{S}}}^{H} \boldsymbol{y} \\
\boldsymbol{A}_{i}^{H} \boldsymbol{y}
\end{array}\right] .
\end{aligned}
$$

Using the block inversion formula [21], we obtain, after some algebraic manipulations,

$$
\begin{aligned}
& \mathbb{E}\left(\boldsymbol{h}_{\mathcal{S}} \mid \boldsymbol{y}, \mathcal{S}\right)= \\
& {\left[\begin{array}{c}
\mathbb{E}\left(\boldsymbol{h}_{\underline{\mathcal{S}}} \mid \boldsymbol{y}, \underline{\mathcal{S}}\right)+\gamma_{\underline{\mathcal{S}}, i}\left(\boldsymbol{u}_{\underline{\mathcal{S}}, i} \boldsymbol{B}_{\underline{\mathcal{S}}, i}^{H} \mathbb{E}\left(\boldsymbol{h}_{\underline{\mathcal{S}}} \boldsymbol{y}, \underline{\mathcal{S}}\right)-\boldsymbol{u}_{\underline{\mathcal{S}}, i} \boldsymbol{q}_{i}\right) \\
\gamma_{\underline{\mathcal{S}}, i}\left(\boldsymbol{q}_{i}-\boldsymbol{B}_{\underline{\mathcal{S}}, i}^{H} \mathbb{E}\left(\boldsymbol{h}_{\underline{\mathcal{S}}} \mid \boldsymbol{y}, \underline{\mathcal{S}}\right)\right)
\end{array}\right],}
\end{aligned}
$$

where

$$
\begin{array}{r}
\boldsymbol{u}_{\underline{\mathcal{S}}, i} \triangleq\left(\boldsymbol{B}_{\underline{\mathcal{S}}, \underline{\mathcal{S}}}\right)^{-1} \boldsymbol{B}_{\underline{\mathcal{S}}, i} \\
\gamma_{\underline{\mathcal{S}}, i} \triangleq \boldsymbol{B}_{i, i}-\boldsymbol{B}_{\underline{\mathcal{S}}, i}^{H} \boldsymbol{u}_{\underline{\mathcal{S}}, i} .
\end{array}
$$

Note that since $\boldsymbol{B}_{i, i}=\eta$ (constant owing to the circulant assumption on $\boldsymbol{A}), \forall i \in\{0, \ldots, N-1\}$, the last equation becomes

$$
\gamma_{\underline{\mathcal{S}}, i}=\eta-\boldsymbol{B}_{\underline{\mathcal{S}}, i}^{H} \boldsymbol{u}_{\underline{\mathcal{S}}, i} .
$$

Eq. (34) is thus a recursive way to calculate $\mathbb{E}\left(\boldsymbol{h}_{\mathcal{S}} \mid \boldsymbol{y}, \mathcal{S}\right)$ that uses the calculated $\mathbb{E}\left(\boldsymbol{h}_{\underline{\mathcal{S}}} \mid \boldsymbol{y}, \underline{\mathcal{S}}\right)$ to obtain a similar calculation for $\mathcal{S}=\underline{\mathcal{S}} \cup\{i\}$.

\section{B. Translational Invariance}

In this subsection, we introduce a further step to reduce the computational complexity involved in calculating the MMSE CIR estimator. First, note that all the computations do not use the elements of the matrix $\boldsymbol{A}$ directly; rather, the elements of the matrix $\boldsymbol{B}=\boldsymbol{A}^{H} \boldsymbol{A}$ are involved. The matrix $\boldsymbol{B}$ can be precomputed and stored. In fact, only the first column of $\boldsymbol{B}$ (i.e., $\boldsymbol{B}_{0}$ ) needs to be known; any other columns, $\boldsymbol{B}_{k}$, can be obtained easily by shifting the elements of $\boldsymbol{B}_{0}$ circularly by $k$ positions. This property of translational invariance, which follows directly from the circulant structure the matrix $B$ inherited from the matrix $\boldsymbol{A}$, also holds for all the quantities in (34) that do not involve the observation vector $\boldsymbol{y}$. Specifically, translational invariance is applicable in the case of both $\boldsymbol{u}_{\underline{\mathcal{S}}, i}$ and $\gamma_{\underline{\mathcal{S}}, i}$ defined in (35). It can easily be shown that for a support set, $\mathcal{X}$, and a column index, $i$, the following pair of relationships hold

$$
\begin{aligned}
& \boldsymbol{u}_{\mathcal{X}, i}=\boldsymbol{u}_{\mathcal{X}+z, i+z} \\
& \gamma_{\mathcal{X}, i}=\gamma_{\mathcal{X}+z, i+z},
\end{aligned}
$$

where $z$ is any integer value and the addition is taken modulo $N$. This means that the values $\boldsymbol{u}_{\underline{\mathcal{S}}, i}$ and $\gamma_{\underline{\mathcal{S}}, i}$ can be reused in the subsequent computations that satisfy (37). In simple terms, if we calculate $\boldsymbol{u}_{\mathcal{X}, i}$ and $\gamma_{\mathcal{X}, i}$, these calculations will remain the same if the cluster $\mathcal{X}$ and the column $i$ are both translated by the same number $z$. This translation can be applied within one cluster, or from one cluster to another.

The complete low-complexity channel estimation algorithm is presented in the following subsection. It is found helpful from implementation view-point to start by processing the largest cluster first, and then 
reuse the computations when the smaller clusters are processed.

\section{Final Estimation Algorithm Summary}

1 pre-compute: $\boldsymbol{B}=\boldsymbol{A}^{H} \boldsymbol{A}, \eta=\boldsymbol{B}_{0,0}$

2 initialize: $p_{b}, \hat{\boldsymbol{h}}=\mathbf{0}, p_{c}=0$

3 compute: $\boldsymbol{q}=\boldsymbol{A}^{H} \boldsymbol{y}, \rho=\boldsymbol{y}^{H} \boldsymbol{y}$

4 for $c=0, \cdots, C-1$

4.1 compute:

$4.2|\mathcal{S}|=1$

4.3 compute: $p(\mathcal{S})$ using $(9)$

4.4 for $k_{1}=0, \cdots,\left|\mathcal{S}_{c}\right|-1$

4.5 compute:

$$
\begin{aligned}
& i=k_{1} \\
& \mathcal{S}=\mathcal{S}_{c}\{i\} \\
& \mathbb{E}\left(\boldsymbol{h}_{\mathcal{S}} \mid \boldsymbol{y}, \mathcal{S}\right)=\frac{\boldsymbol{q}[\mathcal{S}]}{\boldsymbol{B}_{\mathcal{S} S \mathcal{S}}} \\
& p(\boldsymbol{y} \mid \mathcal{S})=-\frac{(\rho-\boldsymbol{q}[\mathcal{S}]) \mathbb{E}\left(\boldsymbol{h}_{\mathcal{S}} \mid \boldsymbol{y}, \mathcal{S}\right)}{2 \sigma_{v}^{2}} \\
& p(\mathcal{S} \mid \boldsymbol{y})=p(\mathcal{S}) p(\boldsymbol{y} \mid \mathcal{S})^{v} \\
& p_{c}=p_{c}+p(\mathcal{S} \mid \boldsymbol{y}) \\
& \hat{\boldsymbol{h}}[\mathcal{S}]=p(\mathcal{S} \mid \boldsymbol{y}) \mathbb{E}\left(\boldsymbol{h}_{\mathcal{S}} \mid \boldsymbol{y}, \mathcal{S}\right) \\
& \underline{\mathcal{S}}=i \\
& |\mathcal{S}|=2 \\
& \text { compute: } p(\mathcal{S}) \text { using }(9) \\
& \text { for } k_{2}=k_{1}+1, \cdots,\left|\mathcal{S}_{c}\right|-1 \\
& \text { compute: } \\
& \text { * } \quad i=\mathcal{S}_{c}\left\{k_{2}\right\} \\
& \text { * } \mathcal{S}=\{\underline{\mathcal{S}}, i\} \\
& \text { * } \quad \boldsymbol{u}_{\underline{\mathcal{S}}, i} \text { and } \gamma_{\underline{\mathcal{S}}, i} \text { using (35) } \\
& \text { * } \quad \mathbb{E}\left(\boldsymbol{h}_{\mathcal{S}} \mid \boldsymbol{y}, \mathcal{S}\right) \text { using (34) } \\
& \text { * } \quad\left\|\boldsymbol{P}_{\mathcal{S}}^{\perp} \boldsymbol{y}\right\|_{2}^{2} \text { using (32) } \\
& \text { * } \quad p(\boldsymbol{y} \mid \mathcal{S}) \text { from }(34) \\
& p(\mathcal{S} \mid \boldsymbol{y})=p(\mathcal{S}) p(\boldsymbol{y} \mid \mathcal{S}) \\
& \text { * } \quad p_{c}=p_{c}+p(\mathcal{S} \mid \boldsymbol{y}) \\
& \text { * } \hat{\boldsymbol{h}}[\mathcal{S}]=\hat{\boldsymbol{h}}[\mathcal{S}] \\
& p(\mathcal{S} \mid \boldsymbol{y}) \mathbb{E}\left(\boldsymbol{h}_{\mathcal{S}} \mid \boldsymbol{y}, \mathcal{S}\right) \\
& \underline{\mathcal{S}}=\mathcal{S} \\
& |\mathcal{S}|=3 \\
& \text { compute: } p(\mathcal{S}) \text { using }(9) \\
& \text { for } k_{3}=k_{2}+1, \cdots,\left|\mathcal{S}_{c}\right|-1 \\
& \text { compute: }
\end{aligned}
$$

$$
\hat{\boldsymbol{h}}\left[0, \cdots,\left|\mathcal{S}_{c}\right|-1\right]=\frac{\begin{array}{c}
\text { Continue until } k_{l}=l \\
p_{c}\left[0, \cdots,\left|\mathcal{S}_{c}\right|-1\right]
\end{array}}{}
$$$$
\cdots
$$

The core of the algorithm is repeating the '*' marked routine for each support set. The process is implemented as a nested for-loop that generates all the combinations of the cluster's possible support sets and updates the values of the quantities required for the MMSE estimator. Note that for the sake of simplicity, the translational invariance feature in (37) is not included in the algorithm description given above. This feature can be implemented by indexing and storing the values of $\boldsymbol{u}_{\underline{\mathcal{S}}, i}$ and $\gamma_{\underline{\mathcal{S}}, i}$ computed each time and reusing them. The above algorithm will, subsequently, be referred to as orthogonal clustering based MMSE (OC-MMSE).

To illustrate the proposed low-complexity method, let us recall Fig. 2, which depicts an example with a $16 \times 16$ measurement matrix and a CIR with two orthogonal clusters. In Fig. 3, a table is provided that lists all the different supports involved in the computation of the MMSE. For each cluster, the computations start with $|\mathcal{S}|=1$. Then the recursions continue to larger support cardinalities. Using recursive computations, for example, the parameters for $\{1,2,3\}$ can be obtained from those of $\{1,2\}$. Support sets falling in the same column, even if they are from different clusters, are translational invariant (for example, $\{2,3\}=\{1,2\}+1$ and $\{10,11,12\}=\{1,2,3\}+9)$. This results in a huge computational saving by reusing previously calculated values. More specifically, the computations for all support sets in a column can be obtained from one set (e.g., the set in bold font) and reused for all the other support sets in the column.

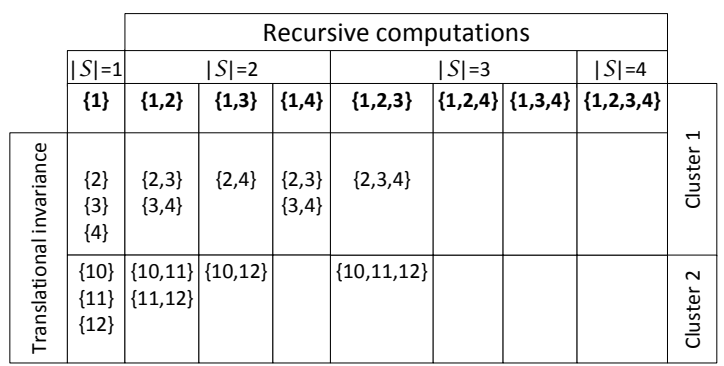

Figure 3: An example for the application of the proposed low-complexity approach to a CIR, $h$, with two orthogonal clusters and a $16 \times 16$ measurement matrix, $\boldsymbol{A}$, as in Fig. 2. The computation batches required to obtain the MMSE estimate of the CIR are listed. The support sets falling in each column (for both $c=0$ and $c=1$ ) satisfy the translational invariance property (37). Hence, the computations for all support sets in a column can be reduced by reusing the computations from one set (e.g., the set in bold font).

\section{Finding The Orthogonal Clusters}

The low-complexity algorithm presented in the preceding section requires that the indices corresponding to each orthogonal cluster of $\boldsymbol{h}$ to be known ${ }^{2}$. In this section, we show how to derive such knowledge from the received signal $\boldsymbol{y}$. In fact, several methods have been proposed in the literature for underwater acoustic signal detection (e.g., see [22]), which can be extended

\footnotetext{
${ }^{2}$ Knowing the clusters is not the same as knowing the support Not all the elements of the clusters are necessarily active. However the first and last elements of a cluster are non zero by definition
} 
to orthogonal cluster detection. However, as the interest is in keeping the computational complexity as low as possible, we propose a simple threshold-based method for signal and then cluster detection. A similar approach is used in $[23,24]$ for finding the first channel tap using a predefined SNR-dependent threshold. The proposed method, however, uses a threshold derived from the noise variance rather than the SNR.

Starting from the signal model in Eq. (2), we propose a simple procedure for detecting the clusters. First let us write (2), in the form

$$
\boldsymbol{y}=\boldsymbol{x}+\boldsymbol{v}=\boldsymbol{A h}+\boldsymbol{v},
$$

where $\boldsymbol{x}$ represents the noiseless version of $\boldsymbol{y}$. Given the cluster-sparse nature of $\boldsymbol{h}$, and assuming that the clusters are sufficiently disjoint, the clustered pattern of $\boldsymbol{h}$ will reflect in both $\boldsymbol{x}$ and $\boldsymbol{y}$ (e.g., see Fig. 7). The support of $\boldsymbol{x}$ could be slightly shifted relative to the support of $\boldsymbol{h}$. However, for a small $L$ (which is the bandwidth of the measurement matrix), the supports of $\boldsymbol{h}$ and $\boldsymbol{x}$ are approximately identical. Therefore, knowing the characteristics of the noise $v$, it can be possible to find the location of the active clusters of $\boldsymbol{h}$ from the observation vector $\boldsymbol{y}$.

Let us consider an element (or sample) of $\boldsymbol{y}$ denoted as $\boldsymbol{y}_{i}=\boldsymbol{x}_{i}+\boldsymbol{v}_{i}$. We want to test if $\boldsymbol{x}_{i}$ is zero or nonzero. This is a binary detection problem, which can be formulated as

$$
\boldsymbol{y}_{i}=b x+\boldsymbol{v}_{i},
$$

where $x$ is a random variable, and $b$ is equal to one when $\boldsymbol{y}_{i}$ is an active element and zero otherwise. To simplify the analysis, let us assume that $\boldsymbol{x}$ has a Gaussian distribution with zero mean and variance $\sigma_{x}^{2}$. On the other hand $\boldsymbol{v}_{i}$ is also a zero-mean Gaussian variable with variance $\sigma_{v}^{2}$. The problem now is to detect the presence of an active signal through the binary variable $b$. A simple way to do so is to apply a positive threshold on the absolute value of $\boldsymbol{y}_{i}$. Thresholding can work perfectly if noise is not present. In this section, it will be shown that even in the presence of noise, thresholding is still capable of detecting active elements with reasonable success rate, especially when the signals are sparse. Now, let us examine the probability

$$
\begin{aligned}
p_{\zeta} & =P\left(\left|\boldsymbol{y}_{i}\right|>\zeta\right)=P\left(\left|b x+\boldsymbol{v}_{i}\right|>\zeta\right) \\
& =P\left(b x+\boldsymbol{v}_{i}<-\zeta\right)+P\left(b x+\boldsymbol{v}_{i}>\zeta\right)
\end{aligned}
$$

which represents the probability of $\left|\boldsymbol{y}_{i}\right|$ exceeding the threshold $\zeta$. Note that $\boldsymbol{y}_{i}$ is zero-mean Gaussian in both cases of $b=0$ and $b=1$. However, the variance in the second case is $\sigma_{x}^{2}+\sigma_{v}^{2}$, compared to $\sigma_{v}^{2}$ in the first case. For a normal distribution with zero mean and variance $\sigma^{2}$, the cumulative distribution function
(CDF) is given by $\Phi\left(\frac{r}{\sigma}\right)$, where $r$ is the distribution value of the random variable and $\Phi(r)$ is given by

$$
\Phi(r)=\frac{1}{2}\left[1+\operatorname{erf}\left(\frac{r}{\sqrt{2}}\right)\right] .
$$

In (41), erf is the error function. After some manipulations using the properties of the normal distribution, Eq. (40) can be written in terms of the CDF (41) as

$$
\begin{aligned}
p_{\zeta}(b) & =2-2 \Phi\left(\frac{\zeta}{\sqrt{2 b \sigma_{x}^{2}+2 \sigma_{v}^{2}}}\right) \\
& =2-2 \Phi\left(\frac{\zeta}{\sqrt{2 b \rho \sigma_{v}^{2}+2 \sigma_{v}^{2}}}\right),
\end{aligned}
$$

where $\rho$ is the SNR defined as $\rho \triangleq \frac{\sigma_{x}^{2}}{\sigma_{v}^{2}}$. Based on (42), $p_{\zeta}(b=1)$ and $p_{\zeta}(b=0)$ represent the probability of detection (PD) and probability of false alarm (PF), respectively.

Now, considering the clustered and sparse nature of the signals, the in-cluster SNR, denoted $\rho_{c}$, will be higher than the overall SNR by a factor equal to the reciprocal of the sparsity rate. More specifically, we have

$$
\rho_{c}=\frac{\rho}{p_{b}} \text {. }
$$

Also, let us express the threshold $\zeta$ in the form $\zeta=$ $\alpha \sigma_{v}$, where $\alpha$ is a positive real value. This, in addition to replacing $\rho$ with $\rho_{c}$ from (43), helps us to write (42) in the form

$$
p_{\zeta}(b)=2-2 \Phi\left(\frac{\alpha}{\sqrt{2} \sqrt{1+b \frac{\rho}{p_{b}}}}\right) .
$$

For feasible support detection, we should minimize the $\mathrm{PF}\left(p_{\zeta}(0)\right)$ while maximizing the $\mathrm{PD}\left(p_{\zeta}(1)\right)$. This can be achieved by proper choice of the parameter $\alpha$. From the definition of $\Phi(r)$ in (41), and as a direct result of the behaviour of the error function, it can easily be shown that $p_{\zeta}(b)$ decreases as $\alpha$ increases, and vice versa. Hence, we end up with a tarde-off; we cannot maximize the PD and minimize the PF simultaneously. Consequently, our task is reduced to judiciously selecting the threshold such that reasonable PD and PF are achieved. We carry out this selection by analyzing (44). Fortunately, from the definition of the $\operatorname{CDF} \Phi(r)$, and due to the way the binary variable $b$ is involved in (44), we always have $p_{\zeta}(b=1)>p_{\zeta}(b=0)$.

For further analysis of (44), Fig. 4 plots $p_{\zeta}(1)$ and $p_{\zeta}(0)$ versus the SNR for different values of $\alpha$ and a sparsity rate $p_{b}=0.1$ (which is a reasonable upper bound on sparsity rate for a truly sparse signal). It can easily be seen that as $\alpha$ increases, both $p_{\zeta}(1)$ and $p_{\zeta}(0)$ decrease. In most cases, $p_{\zeta}(1)$ is much larger than $p_{\zeta}(0)$, which (i.e., $\left.p_{\zeta}(0)\right)$ approaches zero for $\alpha>$ 2. While $p_{\zeta}(1)$ increases substantially with increase in 
the SNR, $p_{\zeta}(0)$ remains constant throughout the SNR range. Based on Fig. 4, a range of values of $\alpha$ can produce reasonable sensitivity while offering low $\mathrm{PF}^{3}$.

The thresholding process ends with labeling each sample of $\boldsymbol{y}$ as "active" or "inactive". The detection of the orthogonal clusters depends on this labeling. Orthogonal clusters can be formed by grouping any pair of active samples that are separated by less than $L$ inactive samples in the same cluster. This results in clusters that are separated by intervals of $L$ inactive samples or more, which makes these clusters mutually orthogonal. A summary of the proposed cluster detection algorithm is given in the following subsection.

\section{A. Cluster Estimation Algorithm Summary}

1) Select a value of $\alpha$ and calculate $\zeta=\alpha \sigma_{v}$.

2) Calculate the binary vector $\boldsymbol{b}$ such that $\boldsymbol{b}_{i}=1$ if $\left|\boldsymbol{y}_{i}\right|>\zeta ; \boldsymbol{b}_{i}=0$ otherwise.

3) Form an orthogonal cluster from any group of consecutive indices, $\mathcal{O}_{c}$, that satisfy the following criteria:

3.1) $\boldsymbol{b}\left[\mathcal{O}_{c}\{0\}\right]=1$ (threshold is exceeded at the first index of the cluster).

3.2) $\boldsymbol{b}\left[\mathcal{O}_{c}\left\{\left|\mathcal{O}_{c}\right|-1\right\}\right]=1$ (threshold is exceeded at the last index of the cluster).

3.3) $\mathbf{0}[L \times 1] \not \subset \boldsymbol{b}\left[\mathcal{O}_{c}\right]$, where $\mathbf{0}[L \times 1]$ is a set of $L$ zeros (make sure that there do not exist $L$, or more, consecutive elements in the cluster where $|\boldsymbol{y}|$ does not exceed the threshold).

\section{B. Threshold Selection}

In our simulations, we apply a threshold value that is sufficiently large to produce a reasonable PD, while keeping PF at a negligible level such that the tendency to pick noise samples as support is minimized. Lowering the PF reduces the redundancy in the detected support, hence saving unnecessary computational cost. The price of minimizing the $\mathrm{PF}$ is, however, reducing the sensitivity of detection, which means a higher probability of missing an active sample, $p_{\text {mis }}=1-p_{\zeta}(1)$. Fortunately, cluster detection, as described above, is a collaborative process that involves multiple samples. Roughly speaking, to miss a whole cluster requires missing at least $L$ consecutive samples of the cluster, an event that can occur with a probability equal to $p_{\text {mis }}^{L}=\left[1-p_{\zeta}(1)\right]^{L}$. Then, again, if $p_{\zeta}(1)$ is reasonably high, cluster detection can be achieved with a good success rate. It is found that for sparsity rates of 0.1 or less, threshold values in the interval 2-4 $\sigma_{v}$ yield excellent cluster detection in most cases.

\footnotetext{
${ }^{3}$ Note that $\alpha$ is not confined to integer values. The values given are just examples.
}

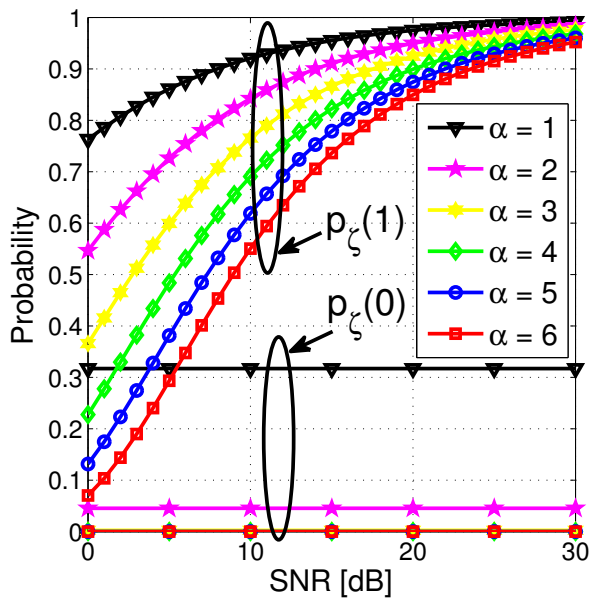

Figure 4: The probabilities, $p_{\zeta}(1)$ and $p_{\zeta}(0)$, versus the SNR plotted for $p_{b}=0.1$ and different threshold levels $\left(\zeta=\alpha \sigma_{v}\right)$.

\section{Simulations AND Results}

The proposed orthogonal clustering based channel estimation method was tested using computer simulations that were carried out using $\mathrm{Matlab}^{\mathrm{TM}}$. The simulation tests were divided into two sets. In the first set, the cluster-sparse channels were generated assuming a Gaussian distribution for the clusters. In the second set, the proposed channel estimation approach was applied to underwater acoustic channels. The purpose of the first set of experiments was to test the proposed method under a simple signal model. The second set of experiments provides an example of a real-world application. Acoustic frequencies were assumed throughout the simulation tests. In all simulation tests, the transmitted probe signal was a Gaussian second derivative pulse with duration of $0.1 \mathrm{~ms}$ and bandwidth of, approximately, $20 \mathrm{kHz}$ (at $-10 \mathrm{~dB}$ ). All the signals were sampled at or above Nyquist rate. Other simulation parameters were $\alpha=3$ and the prior probability $p_{b}$ was set equal to 0.1 . Varying the value of $p_{b}$ within a certain range was found to have no noticeable impact on the results.

The performance of the proposed channel estimation method was benchmarked against a number of compressed sensing methods in addition to the LS channel estimator. The compressed sensing benchmark methods were orthogonal matching pursuit (OMP) [25], fast Bayesian matching pursuit (FBMP) [14,15], and the recent support agnostic Bayesian matching pursuit (SABMP) [16], which uses a greedy approach. Both FBMP and SABMP try to realize an MMSE estimator. FBMP assumes a Gaussian support of the sparse vector, while SABMP is agnostic to the support statistical distribution. SABMP differs from the 
proposed approach in that it performs a greedy search that incorporates only the most probable support sets in the MMSE, and is agnostic to the measurement matrix and sparse vector structures. Our approach, on the other hand, performs an exhaustive evaluation of the MMSE over all possible support sets. A benchmark method is excluded from presentation if it exhibits substantially erroneous results that hinders good visualization of the performance differences between the other methods.

Three metrics were considered for performance evaluation: The normalized mean squared error (NMSE) of the CIR estimate defined as mean $\left[\|(\hat{\boldsymbol{h}}-\boldsymbol{h})\|_{2}^{2} /\|\boldsymbol{h}\|_{2}^{2}\right]$, the symbol error rate (SER) and the computational cost given in terms of the run-time. All the performance metrics were measured at a set of equidistant SNR points. The SNR was defined as the total power contained in the signal samples divided by the total power of the noise samples, with all powers measured at the receiver. In the case where the probe pulse was followed by an OFDM packet, the power calculation included all the samples of the received signal in the probe pulse interval and those in the OFDM packet interval. Performance metrics were calculated over one-thousand independent simulation trials at each SNR point. Each simulation run was performed over a different realization of the CIR.

\section{A. Gaussian Clusters}

In this set of tests, the signals were generated as follows. The CIR consisted of 5 clusters of equal size $(0.1 \mathrm{~ms})$. The cluster real-valued amplitudes were derived from a zero-mean Gaussian distribution with unity variance. The clusters were designed to have an approximate width which was equivalent to that of the underwater acoustic channels examined in the following subsection. The clusters were located in a way that guaranteed perfect orthogonality considering the transmitted probe signal's width. An example of the CIR with the corresponding received signal is shown in Fig. 5. The sampling rate was $40 \mathrm{kHz}$.

Fig. 6 (a) and (b) depict the NMSE and the runtime versus the SNR. It can be seen that the proposed orthogonal clustering based MMSE (OC-MMSE) outperforms all the other methods in the whole SNR range. This superiority is more emphasized in the high SNR regime. The LS and OMP methods preform considerably worse than all the other methods. In fact, the LS and OMP estimators exhibit unreliable performance in most of the cases, as evident by the high ( $>0 \mathrm{~dB}$ ) NMSE. As for computational complexity, the proposed OC-MMSE method also offers the best computational speed. This clearly demonstrates the benefit of orthogonal clustering in reducing computational complexity. Note that OC-MMSE run-time increases with the SNR. This is due to the enhanced sensitivity of the cluster detection algorithm in high SNRs. This results in detection of larger portions of the CIR clusters compared to the low SNR case. Hence, more computations are needed.
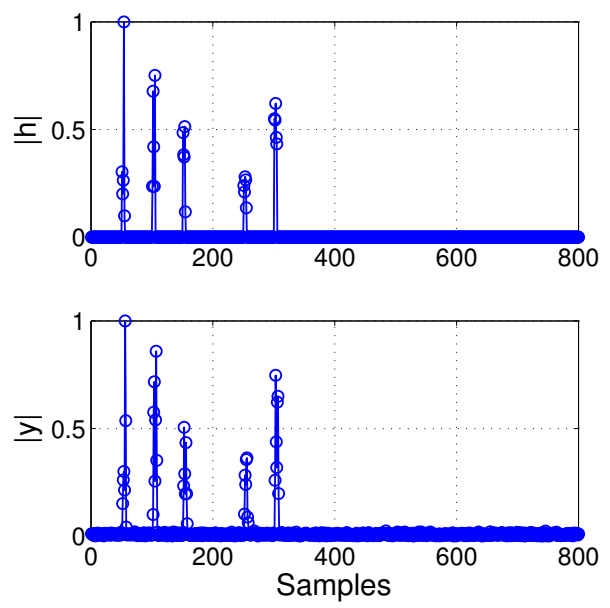

Figure 5: An example of a Gaussian channel impulse response $\boldsymbol{h}$ and the corresponding received signal $\boldsymbol{y}$ contaminated with an AWGN added at 20-dB SNR (normalized magnitudes).

\section{B. Underwater Acoustic Channels}

In this set of tests, the proposed low-complexity cluster-sparse channel estimation method is applied in underwater acoustic communications. Due to the highly variable nature of underwater acoustic environments, it is expected that the cluster-sparse property is not valid in certain cases. In fact, there are many practical cases where the underwater acoustic channels do not follow the cluster-sparse pattern in the exact way required by the proposed method. In addition, the transmission of a short probe pulse, required by the proposed method, may not yield sufficiently accurate channel estimates for adequate symbol detection unless sufficient pulse energy is emitted. The following example tests will consider scenarios with relatively low channel variability and reasonably high probe pulse energy for symbol detection to be carried out successfully.

Underwater acoustic channels were generated based on the statistical models described in [19] using the simulation software package provided in [26]. The package generates complex-valued channels. The simulated scenarios consisted of a transmitter and a receiver located at, respectively, 20-meter and 50-meter depths, with the channel distance being equal to 1000 meters. Fig. 7 shows the magnitude of an exemplar CIR realization together with the received signal $y$ when a $0.1-\mathrm{ms}$ probe pulse is transmitted. In spite of 


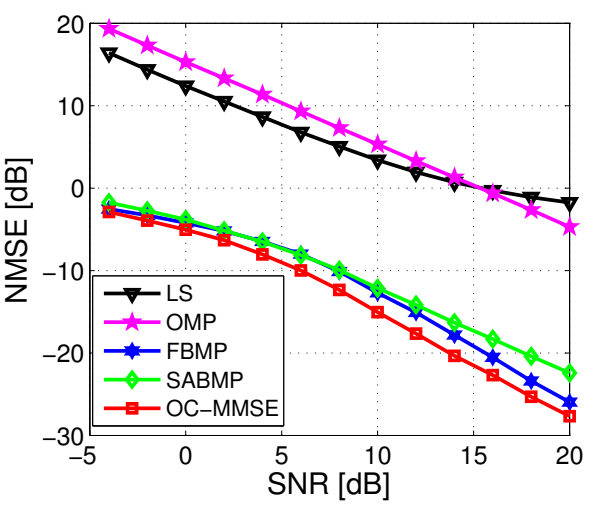

(a)

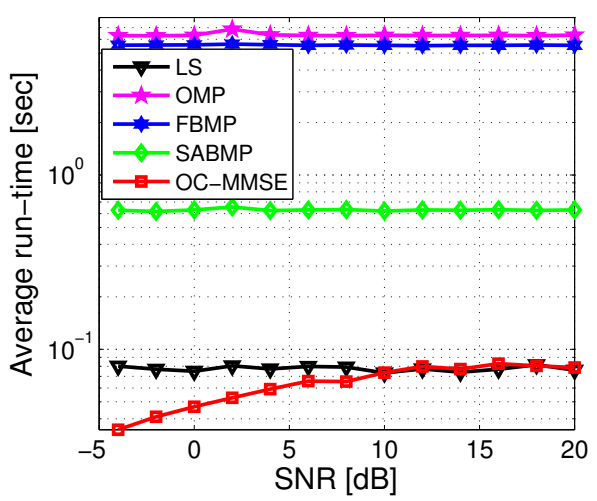

(b)

Figure 6: Performance of the proposed method compared to selected other methods when applied to channels with Gaussian support : a) normalized mean squared error (NMSE); b) average run-time.

the clustered structure, the clusters are not perfectly orthogonal due to some continuity in the CIR that results in clusters which are not completely disjoint. This kind of CIR can be regarded as cluster-sparse only in an approximate sense.

The underwater acoustic channels were generated considering the time-variant nature of these channels. The channels were generated for a period of many tens of seconds with coherence time of 0.7 seconds. The total number of channel realizations generated was 13 thousands. Fig. 8 plots an example of the evolution of the CIR starting from a certain time point. The channels died completely within $20 \mathrm{~ms}$. To simulate the effect of time variability, for each transmitted block of 20-ms duration or more, a CIR vector is assigned (a horizontal slice in Fig. 8). For the next transmitted block, the CIR is changed to the following impulse response in the sequence of generated channels (the next horizontal slice in Fig. 8). Note that the variation of the CIR from a block to the next block is limited, given that the block size is within the coherence time of the channel. This assumption is maintained throughout the simulation tests.

To evaluate the SER, data were transmitted using quadrature amplitude modulation (QAM) over OFDM $[1,27,28]$. Two examples will be discussed. The first example compares the performance of the proposed channel estimation method against the selected benchmark methods when only a probe pulse is used for channel estimation. In the second example, the probe pulse is used only by the proposed OC-MMSE method, while the other techniques are applied to estimate the CIR from pilot frequencies interleaved between the OFDM data. In each example, performance was evaluated using one-thousand simulation trials at each SNR point. In each simulation trial, a pair of adjacent CIR realizations was randomly chosen from the 13thousand realizations available. The NMSE, the average run-time and the SER were used as performance metrics. In these examples of OFDM data transmission, the NMSE was calculated from the frequency-domain representation (namely, the discrete Fourier transform) of the CIRs, where only the frequency bins corresponding to the data subcarriers were involved in calculating the NMSE.
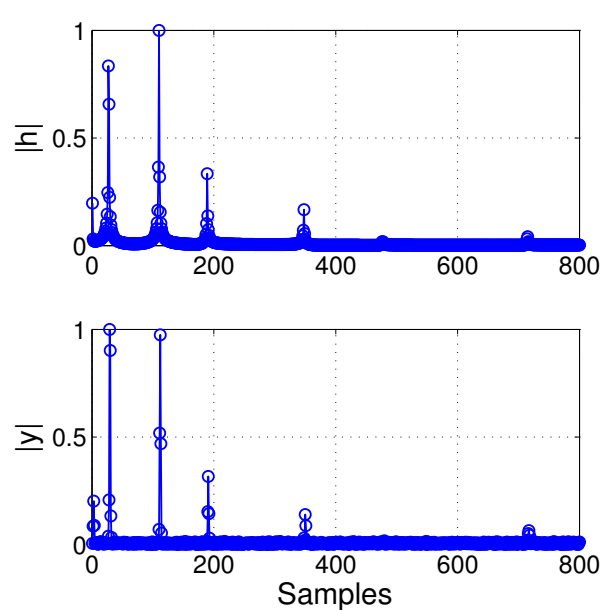

Figure 7: An example of an underwater acoustic channel impulse response $\boldsymbol{h}$ and the corresponding received signal $\boldsymbol{y}$ resulting from a transmitted probe pulse and contaminated with an AWGN at 20-dB SNR (normalized magnitudes).

1) OFDM Example 1: This example compares the performance of different channel estimation techniques when a probe pulse is used as a preamble for OFDM data. The available bandwidth was divided into 1024 subcarriers all of which were used for data transmission using 16-QAM. The transmission bandwidth of the OFDM signals was $20 \mathrm{kHz}$. All signals were generated according to a sampling rate of $80 \mathrm{kHz}$. OFDM packets were generated that consisted of 1024 16-QAM 


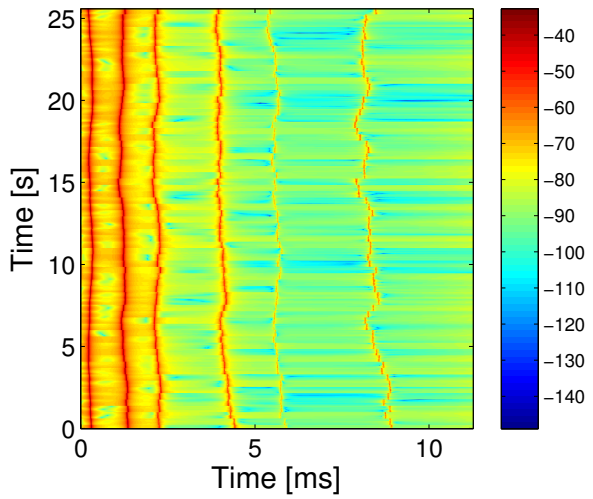

Figure 8: An example of underwater acoustic channel impulse response variation with time.

symbols occurring over a period of 51.2 milliseconds. The packet was followed by a 25.6-millisecond guard interval and preceded by a 0.1-millisecond pulse followed by a 25.5 -millisecond guard interval. This amounts to a symbol transmission rate of $10 \mathrm{ksps}$. The purpose of the guard interval following the OFDM packet was to prevent the multipath pertaining to the OFDM packet from contaminating the subsequent probe pulse. Interference was completely avoided by choosing the guard interval to be longer than the duration of the CIR (which was $20 \mathrm{~ms}$ ). Despite the clear benefit of the guard interval, the downside of such unused time intervals is a reduction in the overall data rate. For instance, in this example, without using the guard interval, a data rate of 13.33 ksps could have been achieved.

The performance of the proposed method and the benchmark methods is depicted in Fig. 9 (a)-(c), where the NMSE, the average run-time and the SER are plotted against the SNR. For each method, the NMSE was calculated as the normalized mean-squared difference between the estimated CIRs and the true probe pulse $C I R$. The LS and OMP methods were removed from these plots due to highly unreliable performance. We observe the following:

- The proposed OC-MMSE clearly outperforms the FBMP and SABMP methods in terms of the NMSE of the channel estimates and average runtime.

- The computation times remain within, approximately, the same ranges compared to those in Fig 6.

- As far as the SER is concerned, the proposed OCMMSE method slightly outperforms the SABMP method. Both methods significantly outperform the FBMP method.

- The SERs of the proposed method stay close to those obtained from the true CIR of the probe (the "Ideal pulse" graph). However, there is a significant deviation from the ideal performance obtained from the true impulse response of the channel through which the OFDM packets were transmitted (the "Ideal OFDM" graph).

- As it appears, for the three algorithms, the differences in soft-decision errors (NMSEs) translate into smaller, yet proportional, differences in harddecision errors (SERs).

2) OFDM Example 2: In this test, the performance of the proposed method, using a probe pulse, is compared to the performance of the benchmark methods, using OFDM pilots which were multiplexed with data. There were two different scenarios. In the first scenario, the transmission consisted of a pulse followed by a guard interval similar to the one in Example 1. The OFDM packet following the guard interval, in this scenario, consisted of 2048 subcarriers used for data transmission. The packet was followed by a guard interval similar to that in Example 1 . In the second scenario, the transmission consisted of similar OFDM packets followed by guard intervals of 25.6-milliseconds. No pulses were used. A quarter (512) of the OFDM subcarriers were assigned as pilot frequencies for channel estimation, while the other 1536 subcarriers were used for data transmission. The pilot subcarriers were chosen such that the subcarrier frequencies were equally spaced, which offered some optimality properties discussed in [29]. In each of the two scenarios, the transmission bandwidth of the OFDM signals was $40 \mathrm{kHz}$ (twice as much as the bandwidth used in Example 1). This resulted in the same subcarrier spacing as in Example 1, while the symbol rate increased to $20 \mathrm{ksps}$. All signals were sampled at a rate of $80 \mathrm{kHz}$. Equal total (pilot plus data) energy was used in both scenarios. The guard intervals in the second scenario were used to avoid intersymbol interference (ISI) between subsequent OFDM packets. ISI was completely avoided by choosing the guard intervals to be longer than the durations of the CIR, hence simplifying the symbol detection process. However, the cost of using these guard intervals was a reduction in the data rate from $30 \mathrm{ksps}$ (without guard intervals) to $20 \mathrm{ksps}$. It is important to note here that since we are simulating the time-variant nature of the acoustic channels, in the first scenario, the pulse goes through a slightly different version of the channel compared to that of the OFDM data. In the second scenario, the OFDM pilots and data were transmitted through the same channel. Thus, in the second scenario, even when the probe channel is estimated perfectly, a deviation from the data channel will occur.

The problem of estimating the CIR from OFDM pilots can be formulated as a linear estimation problem similar to Eq. (2) [27]. The channel estimates obtained 
from the pulse, in the first scenario, and from the pilot OFDM frequencies, in the second scenario, were used for 16-QAM detection. The results are presented in Fig. 10 (a)-(c). For each method, the NMSE was calculated as the normalized mean-squared difference between the estimated CIRs and the true OFDM packet CIR. The NMSE for the LS method was very high; hence, it was removed from the figure to facilitate visualization of the other results. The following points summarize the most pertinent observations:

- The NMSE curves show different trends compared to those shown in Fig. 9 (a). In particular, the NMSEs of the OC-MMSE method seem to be considerably higher in Fig. 10 compared to those in Fig. 9 (a). The main reason for this inconsistency is that the definition of the NMSE in the two figures is different. In Fig. 9 (a), the NMSE was calculated as the normalized meansquared difference between the estimated CIRs and the true probe pulse CIR. On the other hand, in Fig. 10 (a), the NMSE was calculated using the true OFDM packet CIR. Therefore, in Fig. 10, the OC-MMSE is at a disadvantage since the method was applied on the probe pulse signal, while the other methods were applied on OFDM pilot signals.

- The SER plot shows that the OC-MMSE method's SERs, again, stay very close to their ideal expected levels. At low SNRs, this is also close to the ideal performance obtained with the OFDM pilots.

- The FBMP method exhibits the lowest SERs, while the LS and SABMP methods are, in most cases, inferior to both the FBMP and OC-MMSE methods. At the high SNR of $10 \mathrm{dBs}$, the OCMMSE method becomes less efficient and it is outperformed by all the benchmark methods.

- The SER is not as low as one would want it to be. This is normal in underwater acoustic communications. Results can be improved by applying channel codes and deploying multiple receivers [27].

- In terms of computational complexity, the runtime graphs show that the proposed OC-MMSE method provides a very superior computational speed compared to all the other methods. For example, it is found that the proposed method is about 278 times faster than the FBMP method at the SNR of $-10 \mathrm{dBs}$, and 20 times faster at the high SNR of $10 \mathrm{dBs}$. This tremendous difference in computational speed can give the proposed OC-MMSE method a huge advantage for application in situations with computational platforms of limited capabilities.

The results emphasize the robustness of the proposed method, whose performance always stays very close to its ideal expected performance while offering a substantially enhanced computational speed. The merits of the proposed method are mainly attributed to the exhaustive incorporation of the problem structures into the problem solution. Unfortunately, not all these structures are available when OFDM pilots are employed, and hence, the proposed method cannot be applied in such situations.

\section{CONCLUSIONS}

A low-complexity Bayesian channel estimation approach for cluster-sparse channels was presented. The proposed approach capitalized on the structure of the channel impulse response and that of the measurement matrix to realize a computationally efficient, yet more accurate, minimum mean squared error (MMSE) estimator. In the proposed approach, orthogonal clusters were formed by applying a threshold to the received signal vector. Following this step, the realization of the channel MMSE estimator was reduced to parallel cluster-wise computations. Computational complexity was further reduced by reusing computations that satisfied some specific criteria. The proposed method can be applied when the transmission consists of a short probe pulse and the separation between the channel clusters is at least equal to the pulse width. If such conditions are met, the proposed method offers improved mean squared error (MSE) and reduced computational complexity. If the cluster separation is not sufficiently large, or if the channel clusters are not perfectly orthogonal (e.g., the gain of the channel is not exactly zero outside the clusters), the MSE offered by the proposed method will increase.

The performance of the proposed method was evaluated in simulation using channels with Gaussian support amplitudes and also with simulated underwater acoustic channels. From the results it was seen that the proposed method almost always offered the lowest computational complexity compared to selected benchmark methods. When a probe pulse was used by all methods, the results demonstrated the superiority of the proposed method over the benchmark methods in terms of the normalized MSE and the symbol error rate (SER), in addition to the computational speed. When the channels were time variant and the benchmark methods used OFDM pilots multiplexed with the data, while the proposed method used a probe pulse that preceded the data, the SER performance offered by the proposed method was, on average, bettered only by one method. This latter method was more than a hundred time slower than the proposed method, on average. Future work will consider evaluation of the proposed method in a practical experimental setup. 


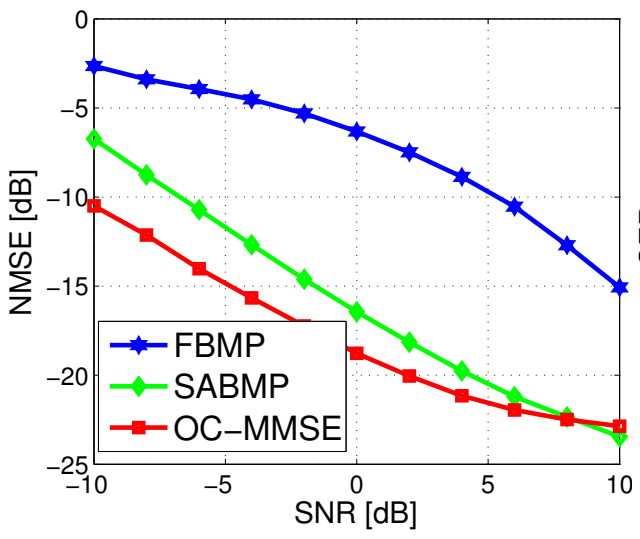

(a)

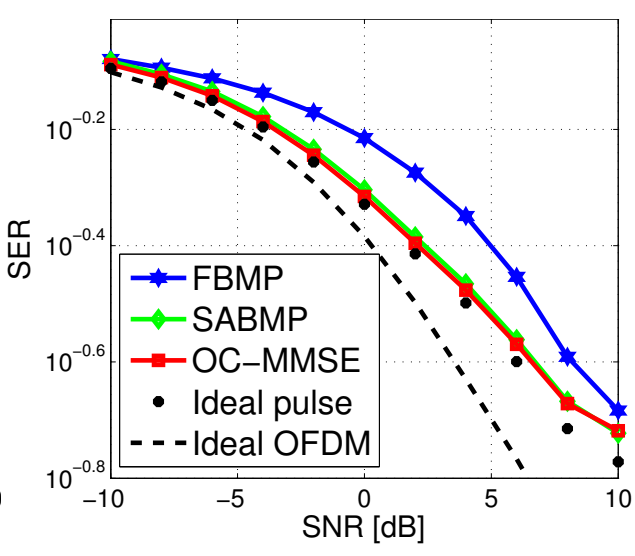

(b)

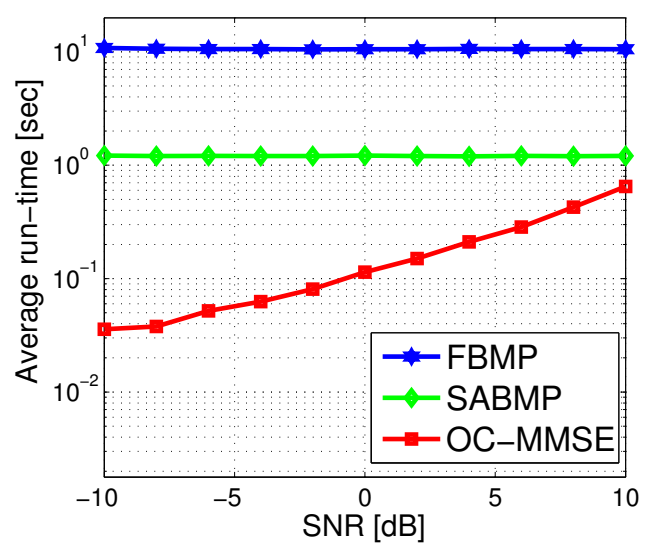

(c)

Figure 9: Performance of the proposed method compared to selected other methods when applied to underwater acoustic channel estimation as in OFDM Example 1: a) normalized mean squared error (NMSE); b) symbol error rate $(\mathrm{SER})$; c) average run-time.

\section{APPENDIX A}

\section{Proof of The Final MMSE Estimator (31)}

Let us consider a simple scenario with only two orthogonal clusters, i.e., $c=0,1$. Any support involved in the sum in (21) can be expressed, based on (22), as $\mathcal{S}=\mathcal{S}_{0} \cup \mathcal{S}_{1}$. For $\mathcal{S} \subseteq \mathcal{O}$; we have $\mathcal{S}_{0} \subseteq \mathcal{O}_{0}$; and $\mathcal{S}_{1} \subseteq \mathcal{O}_{1}$. Therefore, substituting (26), (29) and (30) in (21), we obtain Eq. (A.1). Since the two clusters are independent, (A.1) can be written in the form of Eq. (A.2). Manipulating and replacing both the summation variables $\left(\mathcal{S}_{0}\right.$ and $\left.\mathcal{S}_{1}\right)$ with $\mathcal{S}$, we obtain Eq. (A.3).

Following the same rationale in Eqs. (A.1)-(A.3), when more than two orthogonal clusters are present, we obtain the result in (31).

\section{ACKNOWLEDGMENT}

The authors wish to thank Milica Stojanovic and Parastoo Qarabaqi for making the simulation software package used in this paper available in the public domain, and for clarifying a number of issues related to the channel model in a private communication.

\section{REFERENCES}

[1] M. Morelli and U. Mengali, "A comparison of pilot-aided channel estimation methods for OFDM systems," IEEE Transactions on Signal Processing, vol. 49, no. 12, pp. 3065-3073, Dec 2001.

[2] Olutayo Oyerinde, Channel Estimation for Wireless Communication Systems, Lambert Academic Publishing, 2011.

[3] T. Ballal and T.Y. Al-Naffouri, "Low-sampling-rate ultrawideband channel estimation using equivalent-time sampling," IEEE Transactions on Signal Processing, vol. PP, no. 99, Jul 2014.

[4] Weichang Li and J.C. Preisig, "Estimation of rapidly timevarying sparse channels," IEEE Journal of Oceanic Engineering, vol. 32, no. 4, pp. 927-939, Oct 2007.

[5] AC. Singer, J.K. Nelson, and S.S. Kozat, "Signal processing for underwater acoustic communications," IEEE Communications Magazine, vol. 47, no. 1, pp. 90-96, Jan 2009.

[6] C.R. Berger, Shengli Zhou, J.C. Preisig, and P. Willett, "Sparse channel estimation for multicarrier underwater acoustic communication: From subspace methods to compressed sensing," IEEE Transactions on Signal Processing, vol. 58, no. 3, pp. 1708-1721, Mar 2010. 


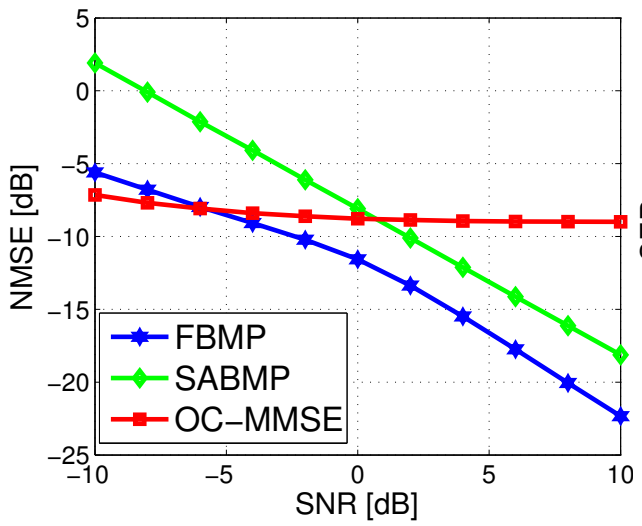

(a)

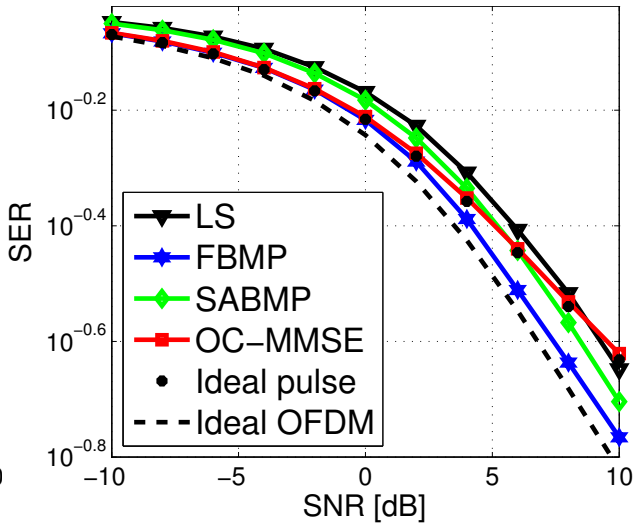

(b)

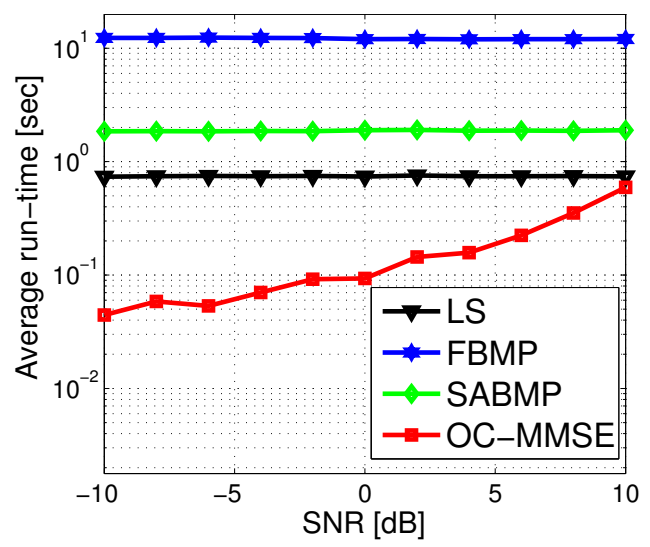

(c)

Figure 10: Performance of the proposed method compared to selected other methods when applied to underwater acoustic channel estimation as in OFDM Example 2: a) normalized mean squared error (NMSE); b) symbol error rate (SER); c) average run-time.

$$
\begin{aligned}
& \hat{\boldsymbol{h}}_{\mathcal{O}}= \frac{\sum_{\mathcal{S}_{0} \subseteq \mathcal{O}_{0}} \sum_{\mathcal{S}_{1} \subseteq \mathcal{O}_{1}} p\left(\boldsymbol{y} \mid \mathcal{S}_{0}\right) p\left(\mathcal{S}_{0}\right) p\left(\boldsymbol{y} \mid \mathcal{S}_{1}\right) p\left(\mathcal{S}_{1}\right)\left[\begin{array}{l}
\mathbb{E}\left(\boldsymbol{h}_{\mathcal{S}_{0}} \mid \boldsymbol{y}, \mathcal{S}_{0}\right) \\
\mathbb{E}\left(\boldsymbol{h}_{\mathcal{S}_{1}} \mid \boldsymbol{y}, \mathcal{S}_{1}\right)
\end{array}\right]}{\sum_{\mathcal{S}_{0} \subseteq \mathcal{O}_{0}} \sum_{\mathcal{S}_{1} \subseteq \mathcal{O}_{1}} p\left(\boldsymbol{y} \mid \mathcal{S}_{0}\right) p\left(\mathcal{S}_{0}\right) p\left(\boldsymbol{y} \mid \mathcal{S}_{1}\right) p\left(\mathcal{S}_{1}\right)} \\
& \hat{\boldsymbol{h}}_{\mathcal{O}}=\frac{\left[\begin{array}{l}
\sum_{\mathcal{S}_{1} \subseteq \mathcal{O}_{1}} p\left(\boldsymbol{y} \mid \mathcal{S}_{1}\right) p\left(\mathcal{S}_{1}\right) \sum_{\mathcal{S}_{0} \subseteq \mathcal{O}_{0}} p\left(\boldsymbol{y} \mid \mathcal{S}_{0}\right) p\left(\mathcal{S}_{0}\right) \mathbb{E}\left(\boldsymbol{h}_{\mathcal{S}_{0}} \mid \boldsymbol{y}, \mathcal{S}_{0}\right) \\
\sum_{\mathcal{S}_{0} \subseteq \mathcal{O}_{0}} p\left(\boldsymbol{y} \mid \mathcal{S}_{0}\right) p\left(\mathcal{S}_{0}\right) \sum_{\mathcal{S}_{1} \subseteq \mathcal{O}_{1}} p\left(\boldsymbol{y} \mid \mathcal{S}_{1}\right) p\left(\mathcal{S}_{1}\right) \mathbb{E}\left(\boldsymbol{h}_{\mathcal{S}_{1}} \mid \boldsymbol{y}, \mathcal{S}_{1}\right)
\end{array}\right]}{\sum_{\mathcal{S}_{0} \subseteq \mathcal{O}_{0}} p\left(\boldsymbol{y} \mid \mathcal{S}_{0}\right) p\left(\mathcal{S}_{0}\right) \sum_{\mathcal{S}_{1} \subseteq \mathcal{O}_{1}} p\left(\boldsymbol{y} \mid \mathcal{S}_{1}\right) p\left(\mathcal{S}_{1}\right)}
\end{aligned}
$$

[7] Chaoran Du, J.S. Thompson, and Y.R. Petillot, "Detector and waveform design for MIMO radar system with noisy channel estimation," Aerospace and Electronic Systems, IEEE Transactions on, vol. 48, no. 3, pp. 2332-2348, Jul 2012.

[8] A Fish, S. Gurevich, R. Hadani, AM. Sayeed, and O. Schwartz, "Delay-Doppler channel estimation in almost linear complexity," IEEE Transactions on Information Theory, vol. 59, no. 11, pp. 7632-7644, Nov 2013.

[9] S. Coleri, M. Ergen, A Puri, and A Bahai, "Channel estimation techniques based on pilot arrangement in OFDM systems," IEEE Transactions on Broadcasting, vol. 48, no. 3, pp. 223229, Sep 2002.

[10] Ming-Xian Chang and Yu-T Su, "Blind and semiblind detections of OFDM signals in fading channels," IEEE Transactions on Communications, vol. 52, no. 5, pp. 744-754, May 2004.
[11] Changyong Shin, R.W. Heath, and E.J. Powers, "Blind channel estimation for MIMO-OFDM systems," IEEE Transactions on Vehicular Technology, vol. 56, no. 2, pp. 670-685, March 2007.

[12] M. Pukkila, Iterative receivers and multi channel equalization for time-division multiple access systems, Dissertation, Helsinki University of Technology Signal Processing Laboratory, 2003.

[13] S. M. Kay, Fundamentals of Statistical Signal Processing, Printice Hall, 1993.

[14] P. Schniter, L.C. Potter, and J. Ziniel, "Fast bayesian matching pursuit," in Information Theory and Applications Workshop, 2008, Jan 2008, pp. 326-333.

[15] P. Schniter, L.C. Potter, and J. Ziniel, "Fast bayesian matching pursuit: Model uncertainty and parameter estimation for sparse linear models," Technical Report, available online 


$$
\hat{\boldsymbol{h}}_{\mathcal{O}}=\left[\begin{array}{l}
\frac{\sum_{\mathcal{S} \subseteq \mathcal{O}_{0}} p(\boldsymbol{y} \mid \mathcal{S}) p(\mathcal{S}) \mathbb{E}\left(\boldsymbol{h}_{\mathcal{S}} \mid \boldsymbol{y}, \mathcal{S}\right)}{\sum_{\mathcal{S} \subseteq \mathcal{O}_{0}} p(\boldsymbol{y} \mid \mathcal{S}) p(\mathcal{S})} \\
\frac{\sum_{\mathcal{S} \subseteq \mathcal{O}_{1}} p(\boldsymbol{y} \mid \mathcal{S}) p(\mathcal{S}) \mathbb{E}\left(\boldsymbol{h}_{\mathcal{S}} \mid \boldsymbol{y}, \mathcal{S}\right)}{\sum_{\mathcal{S} \subseteq \mathcal{O}_{1}} p(\boldsymbol{y} \mid \mathcal{S}) p(\mathcal{S})}
\end{array}\right]
$$

at: http://www2.ece.ohio-state.edu/zinielj/FBMP_TechRep.pdf, 2009.

[16] M. Masood and T.Y. Al-Naffouri, "Sparse reconstruction using distribution agnostic bayesian matching pursuit," IEEE Transactions on Signal Processing, vol. 61, no. 21, pp. 52985309, Nov 2013.

[17] T. Santos, J. Karedal, P. Almers, F. Tufvesson, and AF. Molisch, "Modeling the ultra-wideband outdoor channel: Measurements and parameter extraction method," IEEE Transactions on Wireless Communications, vol. 9, no. 1, pp. 282 290, Jan 2010.

[18] L. Xu G. Gui and L. Shan, "Block Bayesian sparse learning algorithms with application to estimating channels in OFDM systems," in the 17'th International Symposium on Wireless Personal Multimedia Communications (WPMC), 7-10 Sep 2014, Sydney, Australia, pp. 1-5.

[19] P. Qarabaqi and M. Stojanovic, "Statistical characterization and computationally efficient modeling of a class of underwater acoustic channels," IEEE Journal of Oceaninc Engineering, Special Issue: Underwater Communications, vol. 99, no. 4, pp. 701-717, Oct 2013.

[20] M. Chen, "On the solution of circulant linear systems," SIAM Journal on Numerical Analysis, vol. 24, no. 3, pp. 668-683, Jun. 1987.

[21] K. Petersen and M. Pedersen, The Matrix Cookbook, Available online at:, http://www2.imm.dtu.dk/pubdb/p.php?3274, Nov 2012.

[22] D.A. Abraham and P.K. Willett, "Active sonar detection in shallow water using the page test," IEEE Journal of Oceanic Engineering, vol. 27, no. 1, pp. 35-46, Jan 2002.

[23] H. Minn, V.K. Bhargava, and K.B. Letaief, "A robust timing and frequency synchronization for OFDM systems," IEEE Transactions on Wireless Communications, vol. 2, no. 4, pp. 822-839, July 2003.

[24] H. Minn, V.K. Bhargava, and K.B. Letaief, "A combined timing and frequency synchronization and channel estimation for OFDM," in 2004 IEEE International Conference on Communications, June 2004, vol. 2, pp. 872-876.

[25] J.A Tropp and AC. Gilbert, "Signal recovery from random measurements via orthogonal matching pursuit," IEEE Transactions on Information Theory, vol. 53, no. 12, pp. 4655-4666, Dec 2007.

[26] P. Qarabaqi and M. Stojanovic, "Acoustic channel modeling and simulation," available online at: http://millitsa.coe.neu.edu/?q=projects, 2013.

[27] Baosheng Li, Shengli Zhou, M. Stojanovic, L. Freitag, and P. Willett, "Multicarrier communication over underwater acoustic channels with nonuniform Doppler shifts," IEEE Journal of Oceanic Engineering, vol. 33, no. 2, pp. 198-209, Apr 2008.

[28] M. Stojanovic, "OFDM for underwater acoustic communications: Adaptive synchronization and sparse channel estimation," in IEEE International Conference on Acoustics, Speech and Signal Processing, 2008 (ICASSP 2008), Mar 2008, pp. $5288-5291$.

[29] H. Minn and N. Al-Dhahir, "Optimal training signals for MIMO OFDM channel estimation," IEEE Transactions on Wireless Communications, vol. 5, no. 5, pp. 1158-1168, May 2006. 